Archive for

Organic Chemistry

Arkivoc 2019, part ii, 99-113

\title{
gem-Heterosubstituted (stannyl)methylsilanes as synthetic equivalents of functionalized $\alpha$-stannyl(methyl) anions
}

\author{
Damiano Tanini, * Tiziano Nocentini, and Antonella Capperucci* \\ Dipartimento di Chimica "Ugo Schiff", Università di Firenze, Via della Lastruccia 3-13, 50019 Sesto \\ Fiorentino, Italy \\ E-mail: antonella.capperucci@unifi.it
}

Dedicated to Professor Lorenzo Testaferri in the occasion of his $75^{\text {th }}$ birthday

Received 08-02-2019

Accepted 11-03-2019

Published on line 11-30-2019

\section{Abstract}

$\alpha$-Heterosubstituted silyl derivatives, such as phenylthio-, phenylseleno- and benzotriazolyl-stannyl silanes, react with aldehydes under tetra- $n$-butylammonium fluoride (TBAF) catalysis, leading to $\alpha$-substituted- $\beta$ hydroxy stannanes, able to behave as precursors of $Z$ - and E-olefins, generated by deoxystannylation. This reactivity shows the capability of such heterosubstituted silanes to act as masked carbanions through a mild functionalization of the carbon-silicon bond.

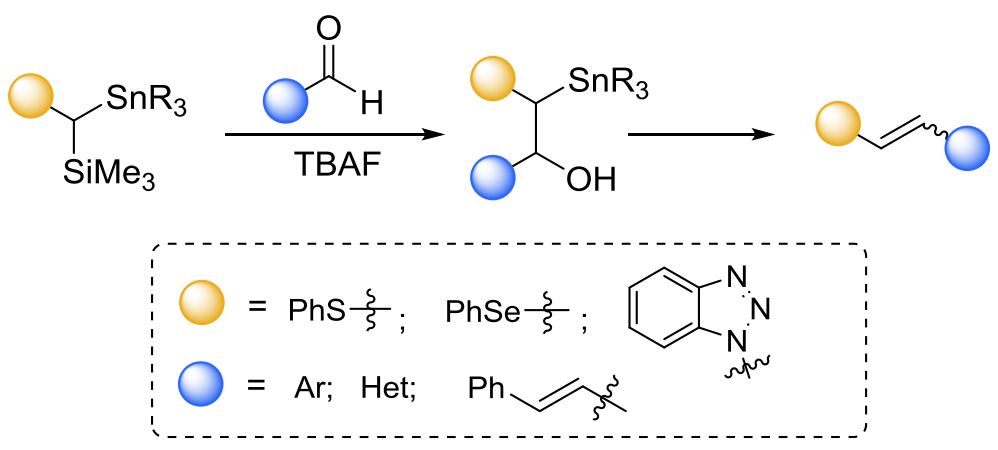

Keywords: Organosilanes, $\beta$-hydroxystannanes, vinyl sulfides, vinyl selenides, fluoride ion, aldehydes 


\section{Introduction}

The chemistry of organosilicon compounds has been studied over the years to search for new synthetic methodologies, able to develop chemical transformations under mild and selective conditions. In this context, their tolerance for various functional groups as well as their application as versatile intermediates in synthetic organic chemistry has been extensively demonstrated. ${ }^{1,2}$ In particular, the fluoride ion activation of a carbonsilicon bond has been commonly used to generate nucleophilic species, under milder conditions with respect to methods based on different organometallic species, such as lithium derivatives. Thus, organosilanes can be used as alternative and efficient reagents compatible with functional groups labile under strong basic conditions. ${ }^{3,4}$ In this context, more versatile compounds containing both a silicon and a tin moiety on $\alpha$ position of heteroatoms may represent interesting structures for different chemical transformations (Figure 1). For instance, $\alpha$-thiosubstituted organosilicon and organotin compounds led to carbanions by transmetallation of the tin moiety with BuLi to afford ketones, ${ }^{5}$ or through deprotonation with LDA (lithium diisopropylamide) or KDA (potassium diisopropylamide) in the presence of hexamethylphosphoramide (HMPA) to afford vinyl stannanes via a Peterson olefination. ${ }^{6} \alpha$-Phenylthio- $\beta$-hydroxystannanes, formed by addition of suitables anions ( $\alpha$-phenylthio(triphenylstannyl)methyllithium or lithium benzenethiolate) with benzaldehyde or epoxides, behaved as intermediates to prepare thio-substituted olefins by deoxystannylation through a tin-Peterson reaction. ${ }^{7-10}$ Heteroatom compounds bearing silicon and tin on the same $\alpha$-carbon were subjected to selective electrochemical oxidation, using silicon and tin as electroauxiliaries, ${ }^{11}$ and then reacted with nucleophiles, showing the versatility of this kind of polyfunctionalized molecules.

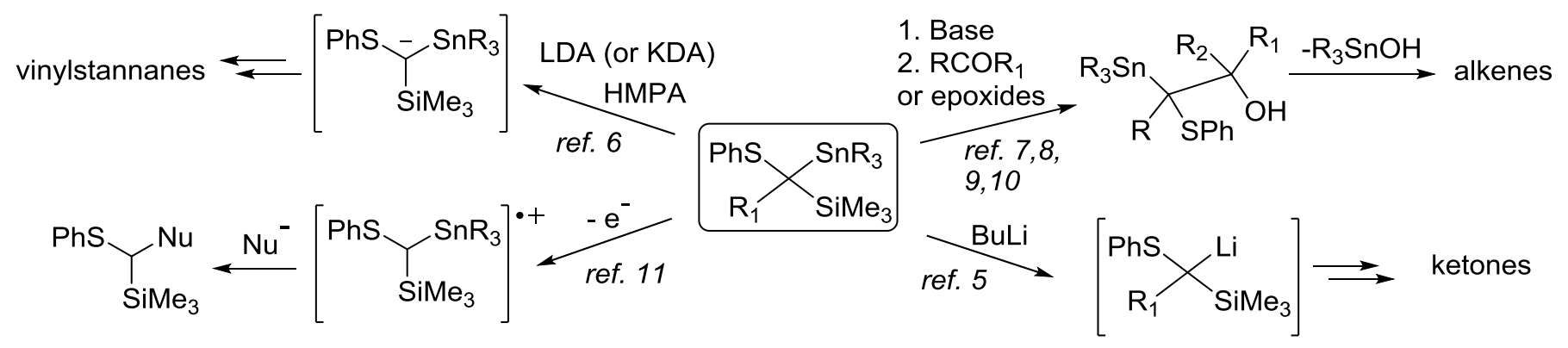

\section{Figure 1}

Our long dated interest in the chemistry of silylated and sulfurated compounds led us to disclose a convenient methodology to transfer linear and heterocyclic $\alpha$-heterosubstituted silyl compounds onto different electrophiles. ${ }^{12}$ (Phenylthiomethyl)trimethylsilane ${ }^{13,14}$ and 2-silylated $1,3-(S, S),-(O, S)$ and $-(N, S)$ five-membered heterocycles ${ }^{12,15}$ could be indeed efficiently functionalized with electrophiles under fluoride ion catalysis. Furthermore, also the seleno derivative (phenylselenomethyl)trimethylsilane was able to react under similar conditions. ${ }^{13}$

Such concepts led us to undertake a systematic investigation on the functionalization on differently $\alpha$ heterosubstituted compounds, such as (phenylthio)-, (phenylseleno)- and benzotriazolyl-stannyl silanes. 


\section{Results and Discussion}

To the best of our knowledge, no direct functionalization of a C-Si bond in the presence of a tin moiety has ever been reported. On the other hand, as described above, phenylthio(methyl)stannanes can be metalated and further reacted with aldehydes to afford the corresponding $\beta$-hydroxy adducts. ${ }^{6,8,9}$

Thus, due to the advantages of the fluoride induced reactivity of the C-Si bond, in terms of mildness and absence of toxic co-solvents like HMPA, we studied the possible selective functionalization of the silicon moiety of [(phenylthio)(stannyl)methyl]silanes 1a-c, bearing different stannylated groups. We initially performed the reaction of 1a with benzaldehyde in the presence of TBAF (tetra- $n$-butylammonium fluoride) (1 eq.) at room temperature (Scheme 1 ).
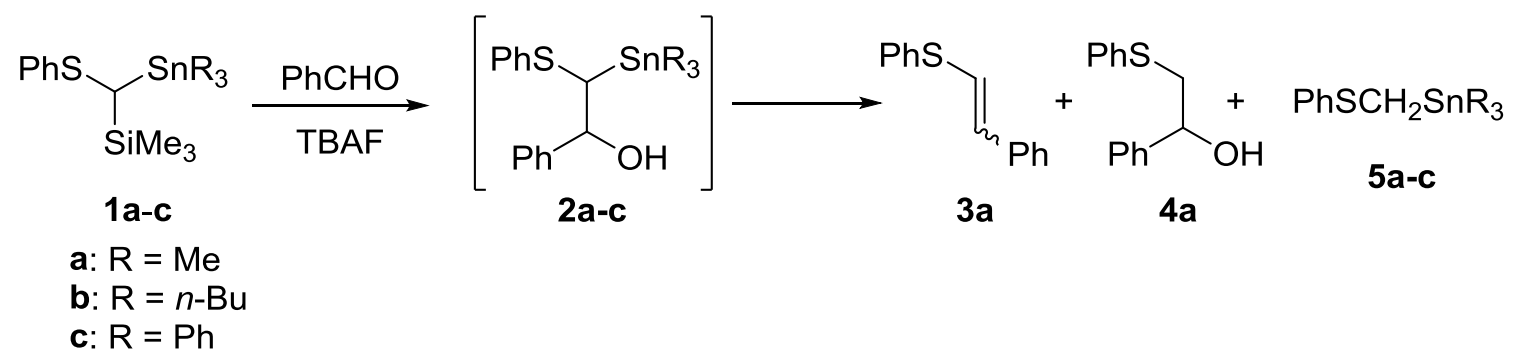

b: $\mathrm{R}=n-\mathrm{Bu}$

c: $\mathrm{R}=\mathrm{Ph}$

Scheme 1. Reactivity of stannyl-silanes 1a-c with benzaldehyde.

The compound 1a reacted quite smoothly under these conditions leading to olefins $\mathbf{3 a}$ (cis:trans 1:2), together with the $\beta$-hydroxy sulfide $\mathbf{4 a}$ and the phenylthio(methyl)stannane $\mathbf{5 a}$, arising from protodesilylation (Scheme 1). To assess the scope of this approach, various aromatic, heteroaromatic and $\alpha, \beta$-unsaturated aldehydes were reacted with 1a-c under the same conditions (Table 1, entries 1-9). In all cases a similar distribution of products was observed. Alkenes 3a-d were always obtained and the trans-isomer was the major compound recovered (cis/trans 1:2 or 1:3, see Table 1). The formation of (phenylthio)methylstannane $\mathbf{5}$ was also observed (ca. 20-30\%, see Table 1). Interestingly, more hindered stannyl derivatives, such as [(phenylthio)(tributylstannyl)methyl]silane 1b and [(phenylthio)(triphenylstannyl)methyl]silane 1c, showed a similar behaviour when treated with different aldehydes under the same conditions (Table 1, entries 5-9).

Although the reaction mixture seemed quite complicated, in our opinion such results were satisfactory, demonstrating the possibility to functionalize stannylsilanes $\mathbf{1}$ under mild conditions. The presence of olefins $\mathbf{3}$ can be indeed attributed to deoxystannylation of $\alpha$-phenylthio- $\beta$-hydroxy stannane intermediates 2 . In fact, olefins arise from stereospecific elimination of $\mathrm{R}_{3} \mathrm{SnOH}$ from adducts 2 , which are obtained as mixture of threo and erythro stereoisomers. Although a syn- or anti-elimination can occur, under the above described basic conditions, and based on the previously reported results, ${ }^{8-10}$ olefins should be formed via a syn-elimination from the less hindered adduct threo-2, affording predominantly $E$-alkenes (Scheme 2). This reaction thus appears as a novel one-pot synthesis of alkenes via the tin-Peterson olefination. 


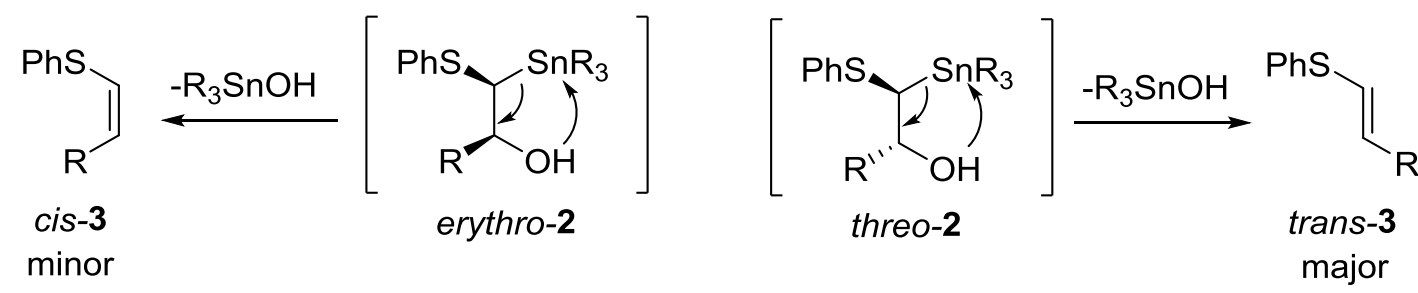

Scheme 2. Formation of olefins $\mathbf{3}$ from $\beta$-hydroxy stannanes $\mathbf{2}$ via syn elimination.

In order to evaluate whether phenylthio- $\beta$-hydroxy stannanes $2 \mathrm{a}-\mathrm{c}$ could be isolated, different reaction conditions were explored. Therefore, we sought to investigate the effect of the amount of TBAF and of the temperature. The tributylstannyl derivative $\mathbf{1 b}$ and $\mathrm{PhCHO}$ were chosen as model substrates and the reaction was initially carried out in the presence of 0.1 eq. of TBAF at ambient temperature. Under these conditions, the formation of the adduct $\mathbf{2 e}$ was evidenced for the first time as mixture of diastereoisomers, although olefins $\mathbf{3 a}$ and products $\mathbf{4}$ and $\mathbf{5}$ were observed in comparable amounts with respect to previous experiments (Table 1, entry 10).

The reaction was then performed at lower temperature $\left(0{ }^{\circ} \mathrm{C}\right)$ and the adduct $2 \mathbf{e}$ was recovered as major product (24\%) (Table 1, entry 11), together with a minor amount of the olefins $\mathbf{3 a}$ (ca $10 \%$ ).

With the aim to verify whether a lower temperature could favour the formation of adducts $\mathbf{2}$, the reaction of the silylstannane $1 \mathrm{~b}$ with $\mathrm{PhCHO}$ was carried out at $-78^{\circ} \mathrm{C}$ in the presence of a catalytic amount of TBAF $(0.1$ eq). Under these conditions, we were pleased to observe the formation of the $\beta$-hydroxystannane $2 \mathrm{e}$ as the major product $(64 \%)$, while only trace amounts of the olefin $\mathbf{3 a}(<10 \%)$ and of $\mathbf{5 b}$ were evidenced (Table 1 , entry 12). The compound $\mathbf{2 e}$ was formed as an almost equimolar mixture of diastereoisomers. It is worth noting that an increased amount of olefins 3 a, whose formation is favoured by the acidity of the medium, was recovered after purification of the crude mixture on silica gel. The reaction was efficiently extended to cinnamaldehyde and thiophene-2-carbaldehyde, enabling the synthesis of the corresponding $\beta$-hydroxy tributylstannanes 2f,g (Table 1, entries 13 and 14), together with minor amounts of olefins $\mathbf{3 b}, \mathbf{d}$ and phenylthio(methyl)stannane $\mathbf{5 b}$. In order to further expand the scope of this study, $\alpha$-silyl stannanes $\mathbf{1 a}$ and 1c, bearing the trimethyl- and the triphenyl-stannyl moieties respectively, were reacted under the same conditions with benzaldehyde. The corresponding adducts $\mathbf{2 a}, \mathbf{h}$ were achieved as predominant compounds (Table 1, entries 15 and 16), with minor amounts of olefins $3 a$. These results confirm that the temperature and the amount of TBAF play a key role in the distribution of the products.

The so obtained results are in line with what reported by other authors on this kind of reactions. In fact, it has been observed ${ }^{8-10}$ that the formation of olefins from $\alpha$-phenylthio- $\beta$-hydroxystannes occurs under acid conditions or by heating. In our procedure, taking advantage of the C-Si functionalization, we may state that room temperature was sufficient to promote the deoxystannylation, thus leading directly to the alkenylsulfides 3 . On the contrary, the reaction performed at low temperatures $\left(0^{\circ} \mathrm{C}\right.$ or $\left.-78^{\circ} \mathrm{C}\right)$ allowed the formation of adducts 2 .

The results obtained in these reactions prompted us to further investigate the generality and the potentialities of the present fluoride ion induced functionalization, and we turned our attention to more intriguing substrates such as $\alpha$-phenylseleno(tributylstannyl)-methyltrimethylsilane 6 , conveniently synthesized from trimethyl-phenylselanylmethyl-silane and tributyltin chloride. Indeed, organoselenium derivatives represent a class of interesting and versatile compounds, finding a wide application both in organic synthesis ${ }^{16-19}$ and in biology. ${ }^{20-22}$ 
Table 1. Influence of the reaction conditions on the transfer of 1a-c onto aldehydes

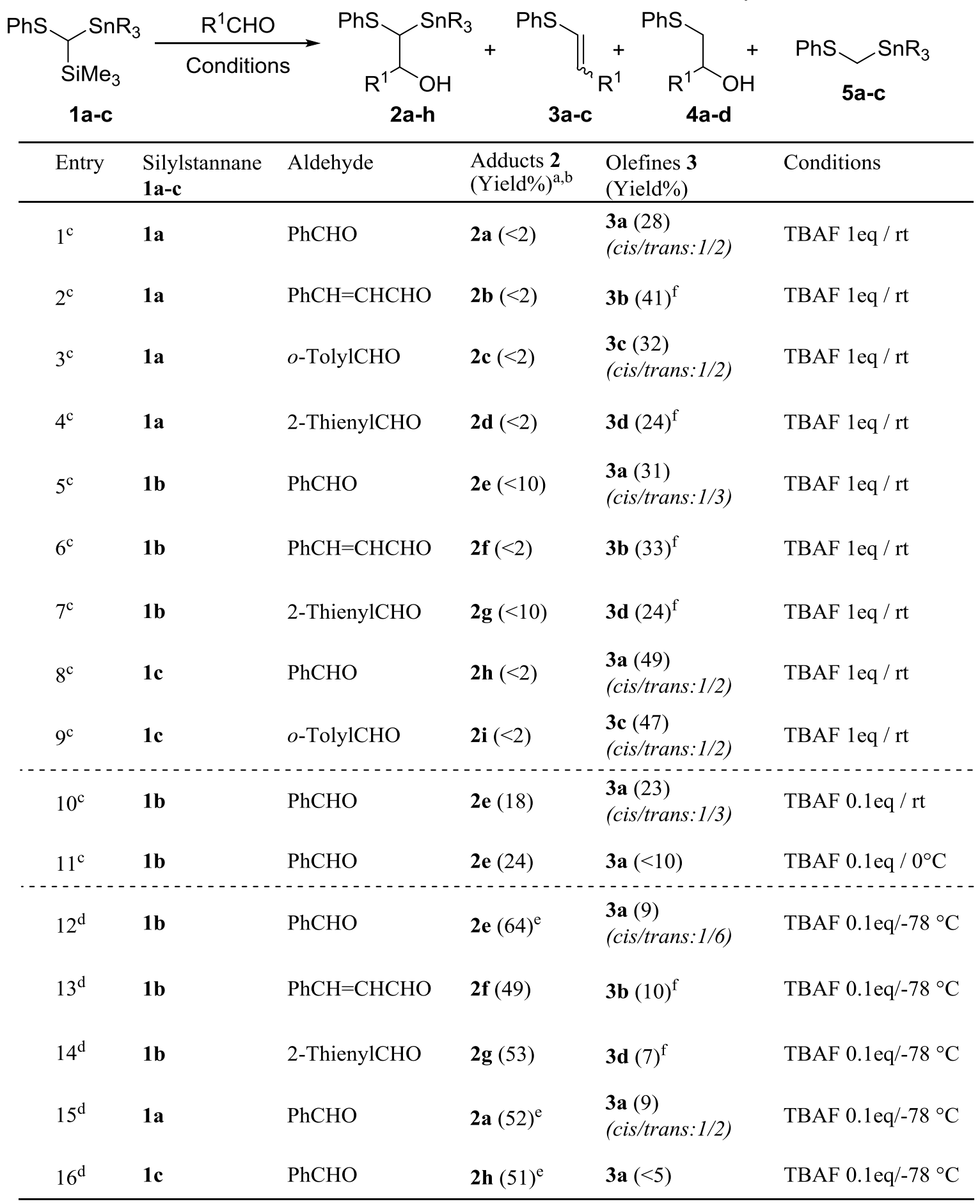

${ }^{a}$ Yield refers to both diastereoisomers. ${ }^{b} 5-15 \%$ of corresponding alcohols 4 was detected. ${ }^{c} 20-30 \%$ of the corresponding desilylated derivatives 5 was detected. ${ }^{d} 5-15 \%$ of the corresponding desilylated derivatives 5 was detected. ${ }^{\mathrm{e}} \mathrm{ca} .10 \%$ of the $-\mathrm{SiMe}_{3}$ protected alcohol was isolated. ${ }^{f}$ cis/trans ratio not determined.

Organoselenides have been used as valuable synthetic intermediates, ligands, and catalysts. Furthermore, a growing number of selenium-containing small molecules has been recently demonstrated to possess an array of biological properties, including antioxidant, ${ }^{23-25}$ anticancer, ${ }^{26}$ and enzyme modulator activities. ${ }^{27-31}$ The $^{2}$ development of novel routes towards organoselenium derivatives is, therefore, highly desirable. ${ }^{16,32}$ In this 
scenario, we recently reported new synthetic procedures for the synthesis of selenium-containing functionalized small molecules under mild conditions. ${ }^{33-35}$

However, despite the interest in the synthesis of organoselenium compounds, to the best of our knowledge, no reports are available on the $\alpha$-metalation of selenyl derivatives. In fact, treatment of selenides with an alkyllithium generally leads to an easy metal-lithium exchange, thus hampering the formation of carbanions, unless suitable substrates are used. ${ }^{36-39}$

Preliminary results ${ }^{40}$ showed that the TBAF-promoted (0.1 eq.) reaction of the selanylated compound 6 with benzaldehyde at room temperature led to the formation of $\alpha$-phenylseleno- $\beta$-hydroxy stannyl derivative 7a, even if in rather low yields, together with the protodesilylated compound $\mathbf{1 0}$ as major product and phenylvinyl selenide 8a, formed through deoxystannylation (Table 2, entry 1). Interestingly, also in the case of the selanylated alkenes, the trans isomer was always the major isomer (cis/trans 2:98), in a higher ratio with respect to vinyl sulfides.

Table 2. Reaction of trimethyl(phenylselanyl(tributylstannyl)methyl)silane 6 with aldehydes

\begin{tabular}{|c|c|c|c|c|}
\hline 6 & & $7 a-c$ & $8 a-c$ & 10 \\
\hline Entry & Aldehyde & $\begin{array}{l}\text { Adduct } 7 \\
\text { (Yield\%) }^{\mathrm{a}, \mathrm{b}}\end{array}$ & $\begin{array}{l}\text { Olefines } 8 \\
\text { (Yield\%) }\end{array}$ & Temperature \\
\hline $1^{\mathrm{c}, \mathrm{d}}$ & $\mathrm{PhCHO}$ & $7 \mathbf{a}(10)$ & $\begin{array}{l}\mathbf{8 a}(15) \\
\text { (cis/trans:2/98) }\end{array}$ & r.t. \\
\hline $2^{\mathrm{c}, \mathrm{e}}$ & $\mathrm{PhCHO}$ & $7 \mathbf{a}(63)$ & $\mathbf{8 a}(<2)$ & $-78^{\circ} \mathrm{C} \rightarrow$ r.t. \\
\hline $3^{\mathrm{c}, \mathrm{e}}$ & 2-ThienylCHO & $7 \mathbf{b}(57)^{\mathrm{f}}$ & $\mathbf{8 b}(<5)^{\mathrm{h}}$ & $-78^{\circ} \mathrm{C} \rightarrow$ r.t. \\
\hline $4^{\mathrm{c}, \mathrm{e}}$ & $\mathrm{PhCH}=\mathrm{CHCHO}$ & $7 c(58)^{\mathrm{g}}$ & $\mathbf{8 c}(7)^{\mathrm{h}}$ & $-78^{\circ} \mathrm{C} \rightarrow$ r.t. \\
\hline
\end{tabular}

${ }^{a}$ Yield refers to both diastereoisomers. ${ }^{b}$ Purification on Florisil. ${ }^{c} 3-10 \%$ of destannylated alcohols $9 a-c$ was detected. ${ }^{d}$ ca. $40 \%$ of the corresponding desilylated derivative 10 was recovered. ${ }^{\mathrm{e}} 10-15 \%$ of the corresponding desilylated derivative 10 was recovered. ${ }^{f}$ d.r. $=9: 1 .{ }^{g}$ d.r. $=1: 1 .{ }^{h}$ cis/trans ratio not determined.

Encouraged by these results, we moved to evaluate the effect of the temperature. Pleasingly, we found that when the silane 6 was treated with benzaldehyde at $-78^{\circ} \mathrm{C}$, the reaction proceeded smoothly affording $\alpha$ phenylseleno- $\beta$-hydroxy stannanes $7 \mathrm{a}$, as major product. The scope of this procedure was enlarged to include differently substituted aldehydes, enabling the formation of selenium-containing $\alpha$-hydroxy stannanes 7a-c, which were isolated in good yields as almost equimolar mixture of diastereoisomers (Table 2, entries 2-4), together with trace amounts of alcohols $\mathbf{9 a - c}$ and the selenide 10. Interestingly, after purification of the crude material on silica gel, olefins $8 \mathbf{a}-\mathbf{c}$ and alcohols $9 a-c$ were recovered as main products. On the contrary, purification on Florisil substantially reduced the deoxystannylation, enabling the isolation of the adducts 7a-c in good yields. 


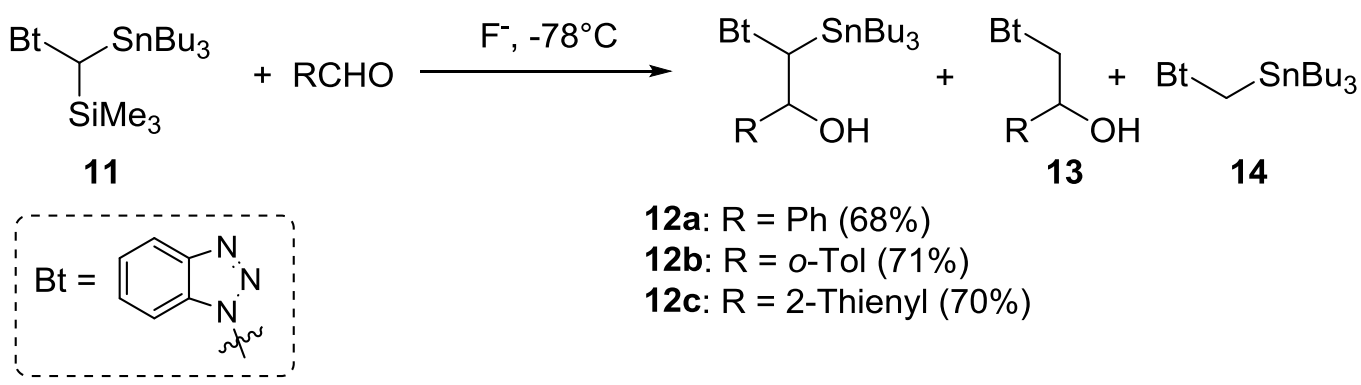

Scheme 3. Functionalization of benzotriazolyl-stannyl-silane $\mathbf{1 1}$ with aldehydes. Yields refer to isolated products. Destannylated alcohols $13 a-c$ were detected in $5-10 \%$ yields.

In order to further explore the scope of this methodology, we synthesized the benzotriazolyl(tributylstannyl)silylmethane $\mathbf{1 1}$ and evaluated its reactivity with aromatic and heteroaromatic aldehydes. Benzotriazolyl derivatives have been demonstrated valuable substrates in organic synthesis and have been widely applied as versatile intermediates. ${ }^{41,42}$ Pleasingly, the desired stannylated derivatives 12a-c were efficiently obtained in good yields through a selective fluoride-induced carbodesilylation reaction (Scheme 3). Amounts of desilylated (benzotriazolyl)stannylmethane $\mathbf{1 4}$ and alcohols 13a-c were recovered as well. Nevertheless, these findings highlight that also this heterosubstituted (stannyl)methylsilane is able to react as efficient nucleophile through a smooth functionalization of the carbon-silicon bond

\section{Conclusions}

The functionalization of a carbon-silicon bond under fluoride ion conditions affords the generation of $\alpha$ heterosubstituted stannyl carbanionic species, able to act as nucleophiles toward different aldehydes leading to the corresponding $\alpha$-functionalized $\beta$-hydroxy stannanes or olefins, depending on the reaction conditions. These results showed that the C-Si bond could be efficiently and selectively functionalized in the presence of a stannyl moiety, as well as of other synthetically useful groups such as phenylthio-, phenylseleno-, and benzotriazolyl- derivatives.

\section{Experimental Section}

General. All reactions were carried out in an oven-dried glassware under inert atmosphere $\left(N_{2}\right)$. Solvents were dried using a solvent purification system (Pure-Solv $\left.{ }^{\mathrm{TM}}\right)$. Flash column chromatography purifications were performed with Silica gel 60 (230-400 mesh). Thin layer chromatography was performed with TLC plates Silica gel $60 \mathrm{~F}_{254}$, which was visualised under UV light, or by staining with an ethanolic acid solution of $p$ anisaldehyde followed by heating. Mass spectra were determined by ionization potential (El, $70 \mathrm{eV}$ ) and by ESI. ${ }^{1} \mathrm{H}$ and ${ }^{13} \mathrm{C}$ NMR spectra were recorded in $\mathrm{CDCl}_{3}$ using a Varian Gemini 200 spectrometers operating at $200 \mathrm{MHz}$ (for ${ }^{1} \mathrm{H}$ ) and $50 \mathrm{MHz}$ (for ${ }^{13} \mathrm{C}$ ). NMR signals were referenced to nondeuterated residual solvent signals (7.26 ppm for ${ }^{1} \mathrm{H}, 77.0 \mathrm{ppm}$ for $\left.{ }^{13} \mathrm{C}\right)$. Chemical shifts $(\delta)$ are given in parts per million (ppm), and coupling constants $(J)$ are given in Hertz $(\mathrm{Hz})$, rounded to the nearest $0.1 \mathrm{~Hz}$. Multiplicity is reported as: $\mathrm{s}=$ singlet, $\mathrm{d}=$ doublet, $\mathrm{t}=$ triplet, ap $\mathrm{d}=$ apparent doublet, $\mathrm{m}=$ multiplet, $\mathrm{dd}=$ doublet of doublet, $\mathrm{bs}=$ broad singlet, $b d=$ broad doublet. Compounds 1a-c were synthesized according to reported procedures. ${ }^{6}$ 
Synthesis of trimethyl-(phenylsulfanyl-trimethylstannanyl-methyl)-silane (1a). A solution of trimethylphenylsulfanylmethyl-silane ( $300 \mathrm{mg}, 310 \mu \mathrm{l}, 1.53 \mathrm{mmol}$ ) in anhydrous THF $(4 \mathrm{~mL})$ at $-78{ }^{\circ} \mathrm{C}$ was treated with 1 $\mathrm{mL}$ of a $1.6 \mathrm{M}$ solution of $n$-BuLi. The reaction was allowed to warm to $0{ }^{\circ} \mathrm{C}$ and stirred at this temperature for $30 \mathrm{~min}$. Then, a solution of trimethyltin chloride $(310 \mathrm{mg}, 1.56 \mathrm{mmol})$ in anhydrous THF (5 mL) was added. The solution was stirred for $4 \mathrm{~h}$ at room temperature and then treated with $2 \mathrm{~mL}$ of a $1 \mathrm{M}$ solution of $\mathrm{NH}_{4} \mathrm{Cl}$. The mixture was extracted with diethyl ether and the organic layers dried over sodium sulfate and evaporated under vacuum to give $1 \mathrm{a}$ as a slightly yellow oil (540 mg, $1.5 \mathrm{mmol}, 98 \%) .{ }^{1} \mathrm{H} \mathrm{NMR}\left(\mathrm{CDCl}_{3}, 200 \mathrm{MHz}\right), \delta(\mathrm{ppm})$ : $0.08(\mathrm{~s}, 9 \mathrm{H}), 0.14(\mathrm{~s}, 9 \mathrm{H}), 1.93(\mathrm{~s}, 1 \mathrm{H}), 7.01-7.29(\mathrm{~m}, 5 \mathrm{H}) .{ }^{13} \mathrm{C} \mathrm{NMR}\left(\mathrm{CDCl}_{3}, 50 \mathrm{MHz}\right), \delta(\mathrm{ppm}):-7.5,-0.1,13.2$, 124.8, 127.5, 128.4, 140.7. MS: $m / z$ (\%) = 360 (2), 195 (31), 167 (100), 135 (47), 74 (87), 74 (99). Elemental analysis: $\mathrm{C}_{13} \mathrm{H}_{24} \mathrm{SSiSn}$ Calcd. $\mathrm{C}, 43.47 ; \mathrm{H}, 6.74$. Found: $\mathrm{C} 43.71 \%, \mathrm{H} 6.51 \%$.

Synthesis of trimethyl-(phenylsulfanyl-triphenylstannanyl-methyl)-silane (1c). A solution of trimethylphenylsulfanylmethyl-silane $(300 \mathrm{mg}, 310 \mu \mathrm{l}, 1.53 \mathrm{mmol})$ in anhydrous THF $(4 \mathrm{~mL})$ at $-78{ }^{\circ} \mathrm{C}$ was treated with $1 \mathrm{~mL}$ of a $1.6 \mathrm{M}$ solution of $n$-BuLi. The reaction was allowed to warm to $0^{\circ} \mathrm{C}$ and stirred at this temperature for $30 \mathrm{~min}$. Then, a solution of triphenyltin chloride $(590 \mathrm{mg}, 1.53 \mathrm{mmol}$ ) in anhydrous THF (5 mL) was added. The solution was stirred for $4 \mathrm{~h}$ at room temperature and then treated with $2 \mathrm{~mL}$ of a $1 \mathrm{M}$ solution of $\mathrm{NH}_{4} \mathrm{Cl}$. The mixture was extracted with diethyl ether and the organic layers dried over sodium sulfate and evaporated under vacuum. The crude material was purified by flash chromatography (petroleum ether/ethyl acetate 10:1) to give $1 \mathrm{c}$ as a viscous oil (493 mg, $0.90 \mathrm{mmol}, 59 \%) .{ }^{1} \mathrm{H} \mathrm{NMR}\left(\mathrm{CDCl}_{3}, 200 \mathrm{MHz}\right), \delta(\mathrm{ppm}):-0.06$ $(\mathrm{s}, 9 \mathrm{H}), 2.67(\mathrm{~s}, 1 \mathrm{H}), 7.00-7.24(\mathrm{~m}, 5 \mathrm{H}), 7.27-7.78(\mathrm{~m}, 15 \mathrm{H}) .{ }^{13} \mathrm{C} \mathrm{NMR}\left(\mathrm{CDCl}_{3}, 50 \mathrm{MHz}\right), \delta(\mathrm{ppm}): 0.1,15.9$, $125.1,127.8,128.4,128.5,128.9,137.1,138.8,140.1$. MS (ESI, positive): $569.2[\mathrm{M}+\mathrm{Na}]^{+}$. Elemental analysis: $\mathrm{C}_{28} \mathrm{H}_{30} \mathrm{SSiSn}$ Calcd. C 61.66\%, H 5.54\%. Found: C 61.43\%, H 5.66\%.

Synthesis of trimethyl-(phenylselanyl-tributylstannanyl-methyl)-silane (6). A solution of trimethylphenylselanylmethyl-silane (236 $\mathrm{mg} 0.98 \mathrm{mmol}$ ) dissolved in anhydrous THF $(2 \mathrm{~mL})$ was added to $2 \mathrm{~mL}$ of a $0.5 \mathrm{M}$ solution of LDA in THF cooled at $-78^{\circ} \mathrm{C}$. The reaction was allowed to warm to $-40^{\circ} \mathrm{C}$ and then stirred for $1 \mathrm{~h}$ at this temperature. Afterwards, tributyltin chloride $(0.3 \mathrm{~mL}, 1.03 \mathrm{mmol})$ was added and the reaction was allowed to warm to room temperature and stirred for additional $4 \mathrm{~h}$. The reaction mixture was then treated with $3 \mathrm{~mL}$ of $1 \mathrm{M} \mathrm{NH}_{4} \mathrm{Cl}$ solution and extracted with diethyl ether $(2 \times 10 \mathrm{~mL}$ ). The organic layer was dried over sodium sulfate and concentrated under vacuum. The crude material was purified by flash chromatography (petroleum ether) to give 6 as a pale orange oil (162 mg, $0.30 \mathrm{mmol}, 31 \%) .{ }^{1} \mathrm{H} \mathrm{NMR}\left(\mathrm{CDCl}_{3}\right.$, $200 \mathrm{MHz}), \delta(\mathrm{ppm}): 0.04(\mathrm{~s}, 9 \mathrm{H}), 0.85-0.98(\mathrm{~m}, 15 \mathrm{H}), 1.21-1.53(\mathrm{~m}, 12 \mathrm{H}), 1.97(\mathrm{~s}, 1 \mathrm{H}), 7.18-7.25(\mathrm{~m}, 3 \mathrm{H}), 7.43-$ $7.48(\mathrm{~m}, 2 \mathrm{H}) .{ }^{13} \mathrm{C} \mathrm{NMR}\left(\mathrm{CDCl}_{3}, 50 \mathrm{MHz}\right), \delta(\mathrm{ppm}): 0.4,11.1,13.6,27.4,29.2,126.0,128.7,131.1,134.6 . \mathrm{MS}:$ m/z (\%) 475 (40), 178 (73), 135 (98), 121 (62), 73 (82), 59 (100). Elemental analysis: $\mathrm{C}_{22} \mathrm{H}_{42}$ SeSiSn Calcd. C, 49.64; H, 7.95. Found: C 49.39\%, H 8.12\%.

Synthesis of 1-((tributylstannyl)(trimethylsilyl)methyl)-1H-benzo[d][1,2,3]triazole (11). $n$-BuLi (3.1 mL of a 1.6 $\mathrm{M}$ solution) was added to a solution of 1-trimethylsilanylmethyl-1H-benzotriazole $(1 \mathrm{~g}, 4.88 \mathrm{mmol})$ in anhydrous THF $(8 \mathrm{~mL})$ at $-78^{\circ} \mathrm{C}$. The reaction colour turned blue. The mixture was maintained under stirring for $1 \mathrm{~h}$ and the temperature was allowed to warm to $0{ }^{\circ} \mathrm{C}$. Afterwards, the mixture was cannulated onto a solution of tributyltin chloride $(1.4 \mathrm{~mL}, 5.17 \mathrm{mmol})$ in THF $(4 \mathrm{~mL})$ cooled at $0^{\circ} \mathrm{C}$. The reaction was stirred at room temperature for $2 \mathrm{~h}$ and then treated with $6 \mathrm{~mL}$ of $1 \mathrm{M} \mathrm{NH}_{4} \mathrm{Cl}$ solution. The mixture was extracted with diethyl ether $(2 \times 20 \mathrm{~mL})$ and the organic layer was dried over sodium sulfate and concentrated under vacuum. The crude material was purified by flash chromatography (petroleum ether/ethyl acetate 8:1) to give 11 as an orange-yellow oil (1.86 g, $3.76 \mathrm{mmol}, 77 \%) .{ }^{1} \mathrm{H} \mathrm{NMR}\left(\mathrm{CDCl}_{3}, 200 \mathrm{MHz}\right), \delta(\mathrm{ppm}): 0.07,(\mathrm{~s}, 9 \mathrm{H})$, $.0 .76-0.83(\mathrm{~m}, 9 \mathrm{H}), 0.88-0.96(\mathrm{~m}, 6 \mathrm{H}), 1.19-1.23(\mathrm{~m}, 12 \mathrm{H}), 3.99(\mathrm{~s}, 1 \mathrm{H}), 7.28-7.43(\mathrm{~m}, 3 \mathrm{H}), 7.97-8.02(\mathrm{~m}, 1 \mathrm{H})$. ${ }^{13} \mathrm{C} \mathrm{NMR}\left(\mathrm{CDCl}_{3}, 50 \mathrm{MHz}\right), \delta$ (ppm): -0.7, 11.4, 13.5, 27.2, 28.8, 37.4, 109.9, 119.8, 123.2, 126.0, 133.5, 145.5. 
MS: m/z (\%) 494 (1), 439 (19), 219 (37), 178 (78), 149 (31), 117 (32), 73 (100). Elemental analysis: $\mathrm{C}_{22} \mathrm{H}_{41} \mathrm{~N}_{3} \mathrm{SiSn}$ Calcd. C, 53.45; $\mathrm{H}, 8.36 ; \mathrm{N}, 9.95$. Found: $\mathrm{C} 53.31 \%, \mathrm{H} 8.49 \%, \mathrm{~N} 10.03$.

General procedure for the synthesis of $\alpha$-phenylthio- $\beta$-hydroxy stannanes (2) (GP1). A THF $1 \mathrm{M}$ solution of stannyl-silane 1 and aldehyde was introduced into a flame dried flask containing activated $4 \mathrm{~A}$ molecular sieves (300 mg). The mixture was cooled at $-78{ }^{\circ} \mathrm{C}$, treated with TBAF ( 0.15 eq. of a $1 \mathrm{M}$ THF solution) and maintained under stirring at this temperature for $1 \mathrm{~h}$. The reaction was then allowed to warm to room temperature and stirred for additional $12 \mathrm{~h}$. Afterwards, the mixture was filtered with diethyl ether through a short pad of $\mathrm{SiO}_{2}$ and the solvent was removed under vacuum. The crude material was purified by flash chromatography (petroleum ether/EtOAc 10:1) to afford the desired compounds 2.

1-Phenyl-2-phenylsulfanyl-2-trimethylstannanyl-ethanol (2a). Following the general procedure GP1, silyl stannyl derivative 1a (53 $\mathrm{mg}, 0.15 \mathrm{mmol}$ ) and benzaldehyde (19 $\mathrm{mg}, 0.18 \mathrm{mmol}$ ) gave, after purification, 2a (colorless oil, $31 \mathrm{mg}, 52 \%)$ as a mixture of diasteroisomers (3:1 d.r.). Diastereoisomer major: ${ }^{1} \mathrm{H} \mathrm{NMR}\left(\mathrm{CDCl}_{3}\right.$, $200 \mathrm{MHz}$ ), $\delta$ (ppm): 0.02 (s, 9H), 3.05 (d, 1H, J $2.2 \mathrm{~Hz}), 3.37$ (d, 1H, J 2.6 Hz), 4.99 (bs, 1H), 7.05-7.41 (10H, m). ${ }^{13} \mathrm{C} \mathrm{NMR}\left(\mathrm{CDCl}_{3}, 50 \mathrm{MHz}\right), \delta$ (ppm): -8.1, 42.0, 73.1, 125.2, 126.6, 127.2, 128.3, 129.1, 129.8, 136.5, 142.5. Diastereoisomer minor: ${ }^{1} \mathrm{H}$ NMR $\left(\mathrm{CDCl}_{3}, 200 \mathrm{MHz}\right), \delta(\mathrm{ppm}):-0.09(\mathrm{~s}, 1 \mathrm{H}), 3.10(\mathrm{~d}, 1 \mathrm{H}, \mathrm{J} 9 \mathrm{~Hz}), 3.28(\mathrm{~d}, 1 \mathrm{H}, \mathrm{J} 2.6$ $\mathrm{Hz}), 4.91(\mathrm{~d}, 1 \mathrm{H}, J 9 \mathrm{~Hz}, 2.6 \mathrm{~Hz}), 7.14-7.43(\mathrm{~m}, 10 \mathrm{H}) .{ }^{13} \mathrm{C} \mathrm{NMR}\left(\mathrm{CDCl}_{3}, 50 \mathrm{MHz}\right), \delta(\mathrm{ppm}):-8.7,41.6,76.7,126.5$, $128.1,128.5,128.6,128.8,130.2,142.3,137.4$. MS (ESI, positive): $394.8[M+H]^{+}$. Elemental analysis: $\mathrm{C}_{17} \mathrm{H}_{22} \mathrm{OSSn}$ Calcd. C, 51.94; $\mathrm{H}, 5.64$. Found: C 51.66\%, H 5.78\%.

1-Phenyl-2-phenylsulfanyl-2-tributylstannanyl-ethanol (2e). Following the general procedure GP1, silyl stannyl derivative $\mathbf{1 b}(921 \mathrm{mg}, 1.9 \mathrm{mmol})$ and benzaldehyde $(242 \mathrm{mg}, 2.3 \mathrm{mmol})$ gave, after purification $2 \mathrm{e}$ (pale yellow oil, $631 \mathrm{mg}, 64 \%$ ) as an equimolar mixture of diastereoisomers. Diastereoisomer A: ${ }^{1} \mathrm{H} N M R$ $\left(\mathrm{CDCl}_{3}, 200 \mathrm{MHz}\right), \delta(\mathrm{ppm}): 0.55-0.96(\mathrm{~m}, 15 \mathrm{H}), 1.14-1.44(\mathrm{~m}, 12 \mathrm{H}), 3.14(\mathrm{~d}, 1 \mathrm{H}, J 3.2 \mathrm{~Hz}), 3.23(\mathrm{~d}, 1 \mathrm{H}, J 8.6 \mathrm{~Hz})$, $4.94(\mathrm{dd}, 1 \mathrm{H}, J 8.2 \mathrm{~Hz}, 3.4 \mathrm{~Hz}), 7.08-7.38(\mathrm{~m}, 10 \mathrm{H}) .{ }^{13} \mathrm{C} \mathrm{NMR}\left(\mathrm{CDCl}_{3}, 50 \mathrm{MHz}\right), \delta(\mathrm{ppm}): 10.3,13.7,27.4,29.0$, 40.6, 76.9, 126.0, 126.5, 127.9, 128.3, 128.7, 129.2, 138.5, 142.7. Diastereoisomer B: ${ }^{1} \mathrm{H} N M R\left(\mathrm{CDCl}_{3}, 200\right.$ $\mathrm{MHz}), \delta(\mathrm{ppm}): 0.67-0.99(\mathrm{~m}, 15 \mathrm{H}), 1.15-1.47(\mathrm{~m}, 12 \mathrm{H}), 3.10(\mathrm{~d}, 1 \mathrm{H}, J 1.8 \mathrm{~Hz}), 3.42(\mathrm{~d}, 1 \mathrm{H}, J 2.2 \mathrm{~Hz}), 4.98(\mathrm{bs}$, $1 \mathrm{H})$, 7.23-7.39 (m, 8H), 7.45-7.50 (m, 2H). ${ }^{13} \mathrm{C} \mathrm{NMR}\left(\mathrm{CDCl}_{3}, 50 \mathrm{MHz}\right), \delta(\mathrm{ppm}): 10.8,13.7,27.4,29.0,41.7$, 73.4, 125.2, 126.5, 127.1, 128.2, 129.0, 129.8, 137.0, 142.7. MS (ESI, positive): $521.4[\mathrm{M}+\mathrm{H}]^{+}$. Elemental analysis: $\mathrm{C}_{26} \mathrm{H}_{40} \mathrm{OSSn}$ Calcd. C, 60.13; $\mathrm{H}, 7.76$. Found: $\mathrm{C} 60.27 \%, \mathrm{H} 7.65 \%$.

(E)-4-Phenyl-1-(phenylthio)-1-(tributylstannyl)but-3-en-2-ol (2f). Following the general procedure GP1, silyl stannyl derivative $\mathbf{1 b}(104 \mathrm{mg}, 0.20 \mathrm{mmol}$ ) and cinnamaldehyde (32 $\mathrm{mg}, 0.24 \mathrm{mmol}$ ) gave $2 \mathrm{f}$ (pale yellow oil, $52 \mathrm{mg}, 48 \%)$ as a mixture of diastereoisomers (3:2 d.r.). Diastereoisomer major: ${ }^{1} \mathrm{H} \mathrm{NMR}\left(\mathrm{CDCl}_{3}, 200 \mathrm{MHz}\right), \delta$ (ppm): 0.81-1.31 (m, 15H), 1.21-1.60 (m, 12H), $2.54(\mathrm{~d}, 1 \mathrm{H}, J 5 \mathrm{~Hz}), 3.24\left(\mathrm{~d}, 1 \mathrm{H}, J 5.4 \mathrm{~Hz}\right.$, coupling with ${ }^{119} \mathrm{Sn}$ $\left.{ }^{2} J_{\text {Sn-H }} 50.8 \mathrm{~Hz}\right), 4.4-4.7(\mathrm{~m}, 1 \mathrm{H}), 6.13(\mathrm{dd}, 1 \mathrm{H}, J 15.8 \mathrm{~Hz}, 7 \mathrm{~Hz}), 6.52(\mathrm{~d}, 1 \mathrm{H}, J 15.8 \mathrm{~Hz}), 7.21-7.54(10 \mathrm{H}, \mathrm{m}) .{ }^{13} \mathrm{C}$ NMR $\left(\mathrm{CDCl}_{3}, 50 \mathrm{MHz}\right), \delta(\mathrm{ppm}): 10.6,13.6,27.4,29.1,38.0,75.1,126.0,126.5,127.5,128.4,128.9,129.2$, 130.4, 131.5, 136.6, 139.1. Diastereoisomer minor: ${ }^{1} \mathrm{H} \mathrm{NMR}\left(\mathrm{CDCl}_{3}, 200 \mathrm{MHz}\right), \delta(\mathrm{ppm}): 2.24(\mathrm{~d}, 1 \mathrm{H}, J 2.6 \mathrm{~Hz})$, $3.26(\mathrm{~d}, 1 \mathrm{H}, J 1.6 \mathrm{~Hz}), 4.57-4.62(1 \mathrm{H}, \mathrm{m}), 6.16(\mathrm{dd}, 1 \mathrm{H}, J 5.4 \mathrm{~Hz}, 16 \mathrm{~Hz}), 6.58$ (dd, $1 \mathrm{H}, J 16 \mathrm{~Hz}, 1.2 \mathrm{~Hz}) . \mathrm{MS}(\mathrm{ESI}$, positive): $546.9[\mathrm{M}+\mathrm{H}]^{+}$. Elemental analysis: $\mathrm{C}_{28} \mathrm{H}_{42} \mathrm{OSSn}$ Calcd. $\mathrm{C}, 61.66 ; \mathrm{H}, 7.76$. Found: $\mathrm{C} 61.41 \%, \mathrm{H} 7.83 \%$.

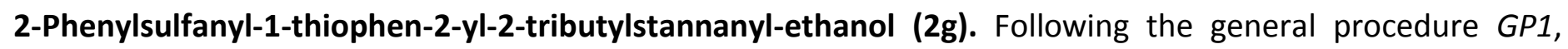
silyl stannyl derivative $\mathbf{1 b}(120 \mathrm{mg}, 0.23 \mathrm{mmol})$ and thiophene-2-carbaldehyde $(27 \mathrm{mg}, 0.28 \mathrm{mmol})$ gave $\mathbf{2 g}$ (yellow oil, $64 \mathrm{mg}, 53 \%) .{ }^{1} \mathrm{H}$ NMR $\left(\mathrm{CDCl}_{3}, 200 \mathrm{MHz}\right), \delta(\mathrm{ppm})$ : 0.62-0.99 (m, 15H), 1.19-1.43 (m, $\left.12 \mathrm{H}\right), 3.19(\mathrm{~d}$, $1 \mathrm{H}, J 3.8 \mathrm{~Hz}), 3.26(\mathrm{~d}, 1 \mathrm{H}, J 7.8 \mathrm{~Hz}), 5.22(\mathrm{dd}, 1 \mathrm{H}, J 7.8 \mathrm{~Hz}, 3.8 \mathrm{~Hz}), 6.86-6.90(\mathrm{~m}, 1 \mathrm{H}), 6.93-6.96(\mathrm{~m}, 1 \mathrm{H}), 7.14-$ $7.28(\mathrm{~m}, 4 \mathrm{H}), 7.36-7.41(\mathrm{~m}, 2 \mathrm{H}) .{ }^{13} \mathrm{C} \mathrm{NMR}\left(\mathrm{CDCl}_{3}, 50 \mathrm{MHz}\right), \delta(\mathrm{ppm}): 10.2,13.7,27.3,29.1,40.9,72.8,124.5$, $129.4,126.2,126.5,128.8,129.4,138.4,146.8$. MS (ESI, positive): $548.6[\mathrm{M}+\mathrm{Na}]^{+}$. Elemental analysis: $\mathrm{C}_{24} \mathrm{H}_{38} \mathrm{OS}_{2} \mathrm{Sn}$ Calcd. C, 54.87; H, 7.29. Found: C 54.65\%, H 7.40\%. 
1-Phenyl-2-phenylsulfanyl-2-triphenylstannanyl-ethanol (2h). Following the general procedure GP1, silyl stannyl derivative 1c (76 mg, $0.14 \mathrm{mmol}$ ) and benzaldehyde (18 $\mathrm{mg}, 1.17 \mathrm{mmol})$ gave, after purification, $2 \mathrm{~h}$ (yellow oil, $41 \mathrm{mg}, 51 \%)$ as an equimolar mixture of diastereoisomers. Diastereoisomer $A:{ }^{1} \mathrm{H} \mathrm{NMR}\left(\mathrm{CDCl}_{3}, 200\right.$ $\mathrm{MHz}), \delta(\mathrm{ppm}): 3.02(\mathrm{~d}, 1 \mathrm{H}, J 2.8 \mathrm{~Hz}), 4.00(\mathrm{~d}, 1 \mathrm{H}, J 3.2 \mathrm{~Hz}), 5.13(\mathrm{bs}, 1 \mathrm{H}), 6.95-7.7(\mathrm{~m}, 25 \mathrm{H}) .{ }^{13} \mathrm{C} \mathrm{NMR}\left(\mathrm{CDCl}_{3}\right.$, $50 \mathrm{MHz}), \delta(\mathrm{ppm}): 44.4,74.7,126.0,126.5,127.6,128.0,128.4,128.5,128.8,129.0,129.9,137.2,138.0$, 142.1. Diastereoisomer $B:{ }^{1} \mathrm{H} N M R\left(\mathrm{CDCl}_{3}, 200 \mathrm{MHz}\right), \delta(\mathrm{ppm}): 3.02(\mathrm{~d}, 1 \mathrm{H}, J 5.2 \mathrm{~Hz}), 3.94(\mathrm{~d}, 1 \mathrm{H}, J 4.8 \mathrm{~Hz})$, 5.16-5.21 (m, $1 \mathrm{H}), 6.95-7.7(\mathrm{~m}, 25 \mathrm{H})$. MS (ESI, positive): $602.5[\mathrm{M}+\mathrm{Na}]^{+}$. Elemental analysis: $\mathrm{C}_{32} \mathrm{H}_{28} \mathrm{OSSn}$ Calcd. C, 66.34; H, 4.87. Found: C 66.21\%, H 4.96\%.

General procedure for the synthesis of olefins (3) (GP2). A THF 1M solution of silane 1 (1.0 eq.) and aldehyde (1.2 eq.) was introduced into a flame dried flask containing activated 4 A molecular sieves (300 $\mathrm{mg}$ ) and then treated with TBAF (1.0 eq. of a $1 \mathrm{M}$ THF solution). The reaction mixture was stirred at room temperature for additional $12 \mathrm{~h}$ and then filtered with diethyl ether through a short pad of $\mathrm{SiO}_{2}$. The solvent was removed under vacuum and the crude material was purified by flash chromatography (petroleum ether/EtOAc 10:1) to afford olefins 3. See Table 1 for isolated yields and d.r. of each product.

Phenyl(styryl)sulfane (3a). Colourless oil. ${ }^{1} \mathrm{H} \mathrm{NMR}\left(\mathrm{CDCl}_{3}, 200 \mathrm{MHz}\right), \delta(\mathrm{ppm}): 6.47(1 \mathrm{H}, \mathrm{d}, J 10.6 \mathrm{~Hz}, \mathrm{Z}$ isomer, minor), $6.57(1 \mathrm{H}, \mathrm{d}, J 10.6 \mathrm{~Hz}, \mathrm{Z}$ isomer, minor), $6.69(1 \mathrm{H}, \mathrm{d}, J 15.8 \mathrm{~Hz}, E$ isomer, major), $6.87(1 \mathrm{H}, \mathrm{d}, J 15.8 \mathrm{~Hz}$, $E$ isomer, major), 7.20-7.38 $(20 \mathrm{H}, \mathrm{m})$. Equimolar mixture of diastereoisomers.

(2-Methylstyryl)(phenyl)sulfane (3c). Yellow oil. ${ }^{1} \mathrm{H} N M R\left(\mathrm{CDCl}_{3}, 200 \mathrm{MHz}\right), \delta(\mathrm{ppm}): 2.33(6 \mathrm{H}, \mathrm{s}), 6.54(1 \mathrm{H}, \mathrm{d}$, $J 10.4 \mathrm{~Hz}, \mathrm{Z}$ isomer, minor), $6.70(1 \mathrm{H}, \mathrm{d}, J 10.4 \mathrm{~Hz}, \mathrm{Z}$ isomer, minor), $6.76(1 \mathrm{H}, \mathrm{d}, J 15.0 \mathrm{~Hz}, E$ isomer, major), $6.98(1 \mathrm{H}, \mathrm{d}, J 15.0 \mathrm{~Hz}, E$ isomer, major), 7.14-7.58 $(18 \mathrm{H}, \mathrm{m})$. Equimolar mixture of diastereoisomers. Spectroscopic data matched those reported in the literature. ${ }^{43,44}$

2-(2-(Phenylthio)vinyl)thiophene (3d). Pale brown oil. ${ }^{1} \mathrm{H} N M R\left(\mathrm{CDCl}_{3}, 200 \mathrm{MHz}\right), \delta$ (ppm): $6.40(1 \mathrm{H}, \mathrm{d}, J 10.8$ $\mathrm{Hz}), 6.70(1 \mathrm{H}, \mathrm{d}, J 15.0 \mathrm{~Hz}), 6.84(1 \mathrm{H}, \mathrm{d}, J 10.8 \mathrm{~Hz}), 6.86(1 \mathrm{H}, \mathrm{d}, J 15.0 \mathrm{~Hz}), 6.91-7.52(16 \mathrm{H}, \mathrm{m})$. Equimolar mixture of diastereoisomers.

1-Phenyl-2-(phenylthio)ethan-1-ol (4a). Pale yellow oil. ${ }^{1} \mathrm{H} N M R\left(\mathrm{CDCl}_{3}, 200 \mathrm{MHz}\right), \delta$ (ppm): 2.88 (1H, bs), $3.04(1 \mathrm{H}, \mathrm{dd}, \mathrm{J}$ 9.5, $13.6 \mathrm{~Hz}), 3.25(1 \mathrm{H}, \mathrm{dd}, \mathrm{J} 3.6,13.6 \mathrm{~Hz}), 4.59-4.72(1 \mathrm{H}, \mathrm{m}), 7.14-7.18(1 \mathrm{H}, \mathrm{m}), 7.17-7.40(7 \mathrm{H}$, $\mathrm{m})$. Spectroscopic data matched those previously reported in the literature. ${ }^{45}$

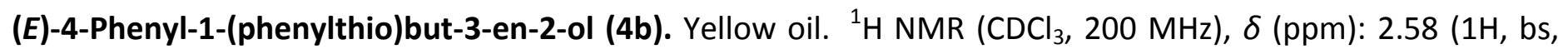
$\mathrm{OH}), 3.04(1 \mathrm{H}, \mathrm{dd}, J$ 8.4, $13.8 \mathrm{~Hz}), 3.26(1 \mathrm{H}, \mathrm{dd}, J 4.0,13.8 \mathrm{~Hz}), 4.32-4.46(1 \mathrm{H}, \mathrm{m}), 6.20(1 \mathrm{H}, \mathrm{dd}, J 6.2,15.8 \mathrm{~Hz})$, $6.64(1 \mathrm{H}, \mathrm{dd}, J 1.0,15.8 \mathrm{~Hz}), 7.18-7.49(10 \mathrm{H}, \mathrm{m})$.

2-(Phenylthio)-1-(thiophen-2-yl)ethan-1-ol (4d). Orange-yellow oil. ${ }^{1} \mathrm{H} \mathrm{NMR}\left(\mathrm{CDCl}_{3}, 200 \mathrm{MHz}\right), \delta(\mathrm{ppm}): 3.0$ $(1 \mathrm{H}, \mathrm{bs}), 3.23(1 \mathrm{H}, \mathrm{dd}, J$ 8.8, $13.8 \mathrm{~Hz}), 3.42(1 \mathrm{H}, \mathrm{dd}, J 4.2,13.8 \mathrm{~Hz}), 5.00(1 \mathrm{H}, \mathrm{dd}, \mathrm{J} 4.2,8.8 \mathrm{~Hz}), 6.90-7.08(2 \mathrm{H}$, $\mathrm{m}), 7.18-7.47(6 \mathrm{H}, \mathrm{m})$.

General procedure for the synthesis of $\alpha$-phenylseleno- $\beta$-hydroxy stannanes (7) (GP3). A THF 1 M solution of stannyl-silane 6 and aldehyde was introduced into a flame dried flask containing activated 4 A molecular sieves $(300 \mathrm{mg})$. The mixture was cooled at $-78{ }^{\circ} \mathrm{C}$, treated with TBAF (0.15 eq. of a $1 \mathrm{M}$ THF solution) and maintained under stirring at this temperature for $1 \mathrm{~h}$. The reaction was then allowed to warm to room temperature and stirred for additional $12 \mathrm{~h}$. Afterwards, the mixture was filtered with diethyl ether through a short pad of Florisil and the solvent was removed under vacuum. The crude material was purified by flash chromatography using Florisil as solid phase and petroleum ether/EtOAc 10:1 as eluent to afford aphenylseleno- $\beta$-hydroxy stannanes 7.

1-Phenyl-2-phenylselanyl-2-tributylstannanyl-ethanol (7a). Following the general procedure GP3, silyl stannyl derivative $6(245 \mathrm{mg}, 0.46 \mathrm{mmol})$ and benzaldehyde (48 $\mathrm{mg}, 0.55 \mathrm{mmol}$ ) gave, after purification, 7a as an orange-yellow oil (164 mg, 63\%) as a mixture of diastereoisomers (3:2 d.r.). Diastereoisomer major: ${ }^{1} \mathrm{H}$ 
NMR $\left(\mathrm{CDCl}_{3}, 200 \mathrm{MHz}\right), \delta(\mathrm{ppm}):$ 0.65-0.73 (m, 6H), 0.82-0.89 (m, 9H), 1.14-1.44 (m, 12H), $3.18(\mathrm{~d}, 1 \mathrm{H}, J 8.6$ $\mathrm{Hz}$, coupling with $\left.{ }^{119} \mathrm{Sn}^{2} \mathrm{~J}_{\mathrm{Sn}-\mathrm{H}} 38.0 \mathrm{~Hz}\right), 3.30(\mathrm{~d}, 1 \mathrm{H}, J 2.8 \mathrm{~Hz}), 4.94(\mathrm{dd}, 1 \mathrm{H}, J 8.6 \mathrm{~Hz}, 2.8 \mathrm{~Hz}), 7.18-7.36(8 \mathrm{H}, \mathrm{m})$, 7.47-7.59 (2H, m). ${ }^{13} \mathrm{C} \mathrm{NMR}\left(\mathrm{CDCl}_{3}, 50 \mathrm{MHz}\right), \delta$ (ppm): 10.3, 13.6, 27.3, 29.0, 38.1, 76.6, 126.5, 127.0, 127.9, 128.4, 128.9, 131.8, 132.8, 142.8. Diastereoisomer minor: ${ }^{1} \mathrm{H} N M R\left(\mathrm{CDCl}_{3}, 200 \mathrm{MHz}\right), \delta(\mathrm{ppm}): 0.71-0.89(\mathrm{~m}$, $15 \mathrm{H}), 1.14-1.45(\mathrm{~m}, 12 \mathrm{H}), 3.06(\mathrm{bs}, 1 \mathrm{H}), 3.44\left(\mathrm{~d}, 1 \mathrm{H}, J 2.8 \mathrm{~Hz}\right.$, coupling with $\left.{ }^{119} \mathrm{Sn}^{2} \mathrm{~J}_{\mathrm{Sn}-\mathrm{H}} 44.2 \mathrm{~Hz}\right), 4.99(\mathrm{~d}, 1 \mathrm{H}, J$ $2.8 \mathrm{~Hz}), 7.20-7.35(\mathrm{~m}, 8 \mathrm{H}), 7.56-7.61(\mathrm{~m}, 2 \mathrm{H}) .{ }^{13} \mathrm{C} \mathrm{NMR}\left(\mathrm{CDCl}_{3}, 50 \mathrm{MHz}\right), \delta(\mathrm{ppm}): 10.8,13.7,14.0,27.3,29.0$, 38.4, 74.4, 125.3, 127.1, 128.2, 129.2, 131.0, 133.0, 142.9. MS: $m / z$ (\%) 260 (35), 179 (79), 165 (24), 102 (85), 91 (26), 77 (100), 51 (51). Elemental analysis: $\mathrm{C}_{26} \mathrm{H}_{40} \mathrm{OSeSn}$ Calcd. C, 55.15; H, 7.12. Found: C 54.92\%, H 7.24\%. 2-Phenylselanyl-1-thiophen-2-yl-2-tributylstannanyl-ethanol (7b). Following the general procedure GP3, silyl stannyl derivative $6(100 \mathrm{mg}, 0.19 \mathrm{mmol})$ and thiophene-2-carbaldehyde $(23 \mathrm{mg}, 0.23 \mathrm{mmol})$ afforded, after purification, $7 \mathbf{b}$ as a pale brown oil $(62 \mathrm{mg}, 57 \%)$ as a mixture of diastereoisomers (5:2 d.r.). Diastereoisomer major: ${ }^{1} \mathrm{H}$ NMR $\left(\mathrm{CDCl}_{3}, 200 \mathrm{MHz}\right), \delta(\mathrm{ppm}): 0.60-0.96(\mathrm{~m}, 15 \mathrm{H}), 1.17-1.48(\mathrm{~m}, 12 \mathrm{H}), 3.22(\mathrm{~d}, 1 \mathrm{H}, J 8.4 \mathrm{~Hz}$, coupling with ${ }^{119} \mathrm{Sn}^{2} J_{\mathrm{Sn}-\mathrm{H}} 49.8 \mathrm{~Hz}$ ), $3.35(\mathrm{~d}, 1 \mathrm{H}, J 3.6 \mathrm{~Hz}), 5.22\left(\mathrm{dd}, 1 \mathrm{H}, J 8.4 \mathrm{~Hz}, 3.6 \mathrm{~Hz}\right.$, , coupling with ${ }^{119} \mathrm{Sn}^{3} J_{\mathrm{Sn}-}$ н 29.0 Hz), 6.85-6.93 (m, 2H), 7.17-7.26 (m, 4H), 7.48-7.54 (m, 2H). ${ }^{13} \mathrm{C} \mathrm{NMR}\left(\mathrm{CDCl}_{3}, 50 \mathrm{MHz}\right), \delta(\mathrm{ppm}): 10.3$, 13.7, 27.4, 29.1, 38.3, 72.6, 124.4, 124.7, 126.5, 127.1, 129.0, 131.8, 132.9, 147.1. Diastereoisomer minor: ${ }^{1} \mathrm{H}$ NMR $\left(\mathrm{CDCl}_{3}, 200 \mathrm{MHz}\right), \delta(\mathrm{ppm}): 0.78-1.01(\mathrm{~m}, 15 \mathrm{H}), 1.17-1.50(\mathrm{~m}, 12 \mathrm{H}), 3.11(\mathrm{~d}, 1 \mathrm{H}, J 3.0 \mathrm{~Hz}), 3.49(\mathrm{~d}, 1 \mathrm{H}, J$ $2.8 \mathrm{~Hz}), 5.21$ (bs, $1 \mathrm{H}), 6.87-6.97(\mathrm{~m}, 2 \mathrm{H}), 7.15-7.35(\mathrm{~m}, 4 \mathrm{H}), 7.51-7.57(\mathrm{~m}, 2 \mathrm{H}) .{ }^{13} \mathrm{C} \mathrm{NMR}\left(\mathrm{CDCl}_{3}, 50 \mathrm{MHz}\right), \delta$ (ppm): 10.7, 13.6, 27.3, 29.0, 37.6, 72.5, 122.9, 124.1, 126.7, 127.2, 129.0, 131.0, 132.5, 147.5. MS: $\mathrm{m} / \mathrm{z}$ (\%) 536 (3), 480 (6), 266 (74), 185 (100). Elemental analysis: $\mathrm{C}_{24} \mathrm{H}_{38} \mathrm{OSSeSn}$ Calcd. C, 50.37; H, 6.69. Found: C $50.11 \%, \mathrm{H} 6.77 \%$.

(E)-4-Phenyl-1-(phenylselanyl)-1-(tributylstannyl)but-3-en-2-ol (7c). Following the general procedure GP3, silyl stannyl derivative $6(100 \mathrm{mg}, 0.19 \mathrm{mmol})$ and cinnamaldehyde $(25 \mathrm{mg}, 0.23 \mathrm{mmol})$ afforded, after purification, 7c as a yellow oil (65 mg, 58\%) as an equimolar mixture of diastereoisomers. Diastereoisomer A: ${ }^{1} \mathrm{H}$ NMR $\left(\mathrm{CDCl}_{3}, 200 \mathrm{MHz}\right), \delta(\mathrm{ppm}):$ 0.83-0.91 (m, 9H), 0.93-1.01 (m, 6H), 1.20-1.55 (m, 12H), $2.67(\mathrm{~d}, 1 \mathrm{H}, J 5.4$ $\mathrm{Hz}), 3.18\left(\mathrm{~d}, 1 \mathrm{H}, J 5.8 \mathrm{~Hz}\right.$, coupling with $\left.{ }^{119} \mathrm{Sn}^{2} \mathrm{~J}_{\mathrm{Sn}-\mathrm{H}} 45.1 \mathrm{~Hz}\right), 4.47-4.63(\mathrm{~m}, 1 \mathrm{H}), 6.12(\mathrm{dd}, 1 \mathrm{H}, J 15.8 \mathrm{~Hz}, 6.6 \mathrm{~Hz})$, $6.52(\mathrm{~d}, 1 \mathrm{H}, J 15.8 \mathrm{~Hz}), 7.12-7.31(\mathrm{~m}, 8 \mathrm{H}), 7.52-7.57(\mathrm{~m}, 2 \mathrm{H}) .{ }^{13} \mathrm{C} \mathrm{NMR}\left(\mathrm{CDCl}_{3}, 50 \mathrm{MHz}\right), \delta(\mathrm{ppm}): 10.7,13.7$, 27.4, 29.2, 35.6, 75.0, 126.4, 126.9, 127.5, 128.3, 129.0, 130.5, 131.8, 132.5, 132.6, 136.5. Diastereoisomer B: ${ }^{1} \mathrm{H}$ NMR $\left(\mathrm{CDCl}_{3}, 200 \mathrm{MHz}\right), \delta(\mathrm{ppm}): 0.83-0.90(\mathrm{~m}, 9 \mathrm{H}), 0.93-1.05(\mathrm{~m}, 6 \mathrm{H}), 1.20-1.54(\mathrm{~m}, 12 \mathrm{H}), 2.72(\mathrm{~d}, 1 \mathrm{H}, J 3.2$ $\mathrm{Hz}), 3.26(\mathrm{~d}, 1 \mathrm{H}, J 2.8 \mathrm{~Hz}), 4.56-4.66(\mathrm{~m}, 1 \mathrm{H}), 6.15(\mathrm{dd}, 1 \mathrm{H}, J 15.8 \mathrm{~Hz}, 5.6 \mathrm{~Hz}), 6.56(\mathrm{dd}, 1 \mathrm{H}, J 15,8 \mathrm{~Hz}, 1.6 \mathrm{~Hz})$, 7.25-7.34 (m, 8H), 7.51-7.59 (m, 2H). $\left.{ }^{13} \mathrm{C} \mathrm{NMR} \mathrm{(CDCl}, 50 \mathrm{MHz}\right), \delta(\mathrm{ppm}):$ 11.04, 13.6, 27.4, 29.1, 36.0, 74.4, 126.4, 127.1, 127.6, 128.5, 129.1, 130.3, 131.0, 132.7,132.8, 136.6. MS: $m / z$ (\%) 389 (2), 286 (53), 205 (84), 177 (18), 128 (100), 76 (38). Elemental analysis: $\mathrm{C}_{28} \mathrm{H}_{42} \mathrm{OSeSn}$ Calcd. C, 56.78; H, 7.15. Found: C 56.95\%, H $7.07 \%$.

(E)-Phenyl(styryl)selane (8a). Viscous pale orange oil. ${ }^{1} \mathrm{H} N M R\left(\mathrm{CDCl}_{3}, 200 \mathrm{MHz}\right), \delta$ (ppm): $6.86(1 \mathrm{H}, \mathrm{d}, \mathrm{J} 15.6$ $\mathrm{Hz}), 7.18(1 \mathrm{H}, \mathrm{d}, J 15.6 \mathrm{~Hz}), 7.20-7.33(8 \mathrm{H}, \mathrm{m}), 7.52-7.58(2 \mathrm{H}, \mathrm{m}) . E$ isomer (d.r. > 98:2). Spectroscopic data matched those reported in the literature. ${ }^{46}$

1-Phenyl-2-(phenylselanyl)ethan-1-ol (9a). Viscous pale brown oil. ${ }^{1} \mathrm{H} N M R\left(\mathrm{CDCl}_{3}, 200 \mathrm{MHz}\right), \delta$ (ppm): 2.80 $(1 \mathrm{H}, \mathrm{bs}, \mathrm{OH}), 3.12(1 \mathrm{H}, \mathrm{dd}, J$ 7.5, $12.3 \mathrm{~Hz}$ ) $, 3.28(1 \mathrm{H}, \mathrm{dd}, J 3.5,12.3 \mathrm{~Hz}), 4.75(1 \mathrm{H}, \mathrm{dd}, J 3.5,7.5 \mathrm{~Hz}), 7.25-7.28$ $(5 \mathrm{H}, \mathrm{m})$. Spectroscopic data matched those reported in the literature. ${ }^{47}$

General procedure for the synthesis of $\alpha$-benzotriazolyl- $\beta$-hydroxy stannanes (12) (GP4). A THF 1M solution of stannyl-silane 11 and aldehyde was introduced into a flame dried flask containing activated $4 \mathrm{~A}$ molecular sieves (300 mg). The mixture was cooled at $-78{ }^{\circ} \mathrm{C}$, treated with TBAF (0.15 eq. of a $1 \mathrm{M}$ THF solution) and maintained under stirring at this temperature for $1 \mathrm{~h}$. The reaction was then allowed to warm to room temperature and stirred for additional $12 \mathrm{~h}$. Afterwards, the mixture was filtered with diethyl ether through a 
short pad of $\mathrm{SiO}_{2}$ and the solvent was removed under vacuum. The crude material was purified by flash chromatography (petroleum ether/EtOAc 10:1) to afford the desired compounds 12.

2-Benzotriazol-1-yl-1-phenyl-2-tributylstannanyl-ethanol (12a). Following the general procedure GP4, silyl stannyl derivative 11 (263 $\mathrm{mg} 0.53 \mathrm{mmol}$ ) and benzaldehyde (68 mg, $0.64 \mathrm{mmol}$ ) afforded, after purification, $12 \mathrm{a}$ as a slighthly yellowish oil $(190 \mathrm{mg}, 68 \%)$ as a mixture of diastereoisomers (5:2 d.r.), together with a minor amount (ca. 10\%) of the corresponding trimethylsilyl ethers 15. Diastereoisomer major: ${ }^{1} \mathrm{H} \mathrm{NMR}\left(\mathrm{CDCl}_{3}\right.$, $200 \mathrm{MHz}), \delta(\mathrm{ppm}): 0.74-0.88(\mathrm{~m}, 15 \mathrm{H}), 1.07-1.39(\mathrm{~m}, 12 \mathrm{H}), 3.02(\mathrm{bd}, 1 \mathrm{H}, J 4.8 \mathrm{~Hz}), 4.71(\mathrm{~d}, 1 \mathrm{H}, J 5.8 \mathrm{~Hz}$, coupling with $\left.{ }^{119} \mathrm{Sn}:{ }^{2} \mathrm{~J}_{\mathrm{Sn}-\mathrm{H}} 38 \mathrm{~Hz}\right), 5.38\left(\mathrm{dd}, 1 \mathrm{H}, J 5.8 \mathrm{~Hz}, 2.2 \mathrm{~Hz}\right.$, coupling with $\left.{ }^{119} \mathrm{Sn}:{ }^{3} \mathrm{~J}_{\mathrm{Sn}-\mathrm{H}} 8 \mathrm{~Hz}\right), 7.13-7.44(\mathrm{~m}$, $8 \mathrm{H}), 7.97-8.01(\mathrm{~m}, 1 \mathrm{H}) .{ }^{13} \mathrm{C} \mathrm{NMR}\left(\mathrm{CDCl}_{3}, 50 \mathrm{MHz}\right), \delta(\mathrm{ppm}):$ 11.4, 13.5, 27.2, 28.8, 57.4, 77.0, 109.6, 119.8, 123.6, 125.5, 126.8, 128.0, 128.5, 133.3, 142.0, 145.6. Diastereoisomer minor: ${ }^{1} \mathrm{H} \mathrm{NMR}\left(\mathrm{CDCl}_{3}, 200 \mathrm{MHz}\right), \delta$ (ppm): 0.69-0.95 (m, 15H), 1.05-1.43 (m, 12H), 3.04 (bs, $1 \mathrm{H}), 4.71\left(\mathrm{~d}, 1 \mathrm{H}, J 8.2 \mathrm{~Hz}\right.$, coupling with ${ }^{119} \mathrm{Sn:}{ }^{2} \mathrm{~J}_{\mathrm{Sn}-\mathrm{H}} 37$ $\mathrm{Hz}$ ), 5.34 (dd, $1 \mathrm{H}, J 8.2 \mathrm{~Hz}, 4.8 \mathrm{~Hz}$, coupling with $\left.{ }^{119} \mathrm{Sn}:{ }^{3} \mathrm{~J}_{\mathrm{Sn}-\mathrm{H}} 37 \mathrm{~Hz}\right), 7.21-7.46(\mathrm{~m}, 8 \mathrm{H}), 7.85-7.90(\mathrm{~m}, 1 \mathrm{H}) . \mathrm{MS}$ (ESI, positive): $552.0[\mathrm{M}+\mathrm{Na}]^{+}$. Elemental analysis: $\mathrm{C}_{26} \mathrm{H}_{39} \mathrm{~N}_{3} \mathrm{OSn}$ Calcd. C, 59.11; $\mathrm{H}, 7.44 ; \mathrm{N}, 7.95$. Found: $\mathrm{C}$ 58.93\%, H 7.60\%, N 8.07. Silyl ether 15 (diastereoisomer $A):{ }^{1} \mathrm{H} N M R\left(\mathrm{CDCl}_{3}, 50 \mathrm{MHz}\right), \delta(\mathrm{ppm}):-0.54(\mathrm{~s}, 9 \mathrm{H})$, 0.45-0.70 (m, 6H), 0.70-0.82 (m, 9H), 1.00-1.4 (m, 12H), $4.59\left(\mathrm{~d}, 1 \mathrm{H}, J 10.2 \mathrm{~Hz}\right.$, coupling with ${ }^{119} \mathrm{Sn}:{ }^{2} \mathrm{~J}_{\mathrm{Sn}-\mathrm{H}} 40$ $\mathrm{Hz}), 5.12\left(\mathrm{~d}, 1 \mathrm{H}, J 10.2 \mathrm{~Hz}\right.$, coupling with $\left.{ }^{119} \mathrm{Sn}:{ }^{3} \mathrm{~J}_{\mathrm{Sn}-\mathrm{H}} 8 \mathrm{~Hz}\right), 7.27-7.49(\mathrm{~m}, 8 \mathrm{H}), 7.98-8.02(\mathrm{~m}, 1 \mathrm{H})$. Silyl ether 15 (diastereoisomer B): ${ }^{1} \mathrm{H}$ NMR $\delta:-0.18(\mathrm{~s}, 9 \mathrm{H}), 0.7-1.0(\mathrm{~m}, 15 \mathrm{H}), 1.1-1.45(\mathrm{~m}, 12 \mathrm{H}), 4.67(\mathrm{~d}, 1 \mathrm{H}, J 6.6 \mathrm{~Hz}$, coupling with $\left.{ }^{119} \mathrm{Sn}:{ }^{2} \mathrm{~J}_{\mathrm{Sn}-\mathrm{H}} 40 \mathrm{~Hz}\right), 5.24\left(\mathrm{~d}, 1 \mathrm{H}, J 6.6 \mathrm{~Hz}\right.$, coupling with $\left.{ }^{119} \mathrm{Sn}:{ }^{3} \mathrm{~J}_{\mathrm{Sn}-\mathrm{H}} 38 \mathrm{~Hz}\right), 7.07-7.31(\mathrm{~m}, 8 \mathrm{H})$, 7.91-7.99 (m, 1H). ${ }^{13} \mathrm{C} \mathrm{NMR}\left(\mathrm{CDCl}_{3}, 50 \mathrm{MHz}\right), \delta(\mathrm{ppm}):-0.1,11.5,13.6,26.8,28.8,58.1,109.8,119.7,123.2$, $126.0,126.3,127.7,128.3,133.6,142.9,145.8 . \mathrm{MS}$ (ESI, positive): $601.8[\mathrm{M}+\mathrm{H}]^{+}$.

2-Benzotriazol-1-yl-1-o-tolyl-2-tributylstannanyl-ethanol (12b). Following the general procedure GP4, silyl stannyl derivative 11 (140 mg, $0.28 \mathrm{mmol}$ ) and 2-methyl-benzaldehyde (41 mg, $0.34 \mathrm{mmol}$ ) afforded 12b as as a yellow oil (108 mg, 71\%) as an equimolar mixture of diastereoisomers. Diastereoisomer $A:{ }^{1} \mathrm{H} N M R$ $\left(\mathrm{CDCl}_{3}, 200 \mathrm{MHz}\right), \delta(\mathrm{ppm}):$ 0.7-0.95 $(\mathrm{m}, 15 \mathrm{H}), 1.1-1.5(\mathrm{~m}, 12 \mathrm{H}), 2.01(\mathrm{~s}, 3 \mathrm{H}), 2.95(\mathrm{~d}, 1 \mathrm{H}, J 1.8 \mathrm{~Hz}), 4.74(\mathrm{~d}, 1 \mathrm{H}$, J $5.6 \mathrm{~Hz}$, coupling with $\left.{ }^{119} \mathrm{Sn}:{ }^{2} \mathrm{~J}_{\mathrm{Sn}-\mathrm{H}} 39 \mathrm{~Hz}\right), 5.48\left(\mathrm{dd}, 1 \mathrm{H}, J 5.6 \mathrm{~Hz}, 1.8 \mathrm{~Hz}\right.$, coupling with $\left.{ }^{119} \mathrm{Sn}^{3} \mathrm{~J}_{\mathrm{Sn}-\mathrm{H}} 41 \mathrm{~Hz}\right), 6.65-$ $6.98(\mathrm{~m}, 1 \mathrm{H}), 7.07-7.32(\mathrm{~m}, 5 \mathrm{H}), 7.67-7.72(\mathrm{~m}, 1 \mathrm{H}), 7.96-8.01(\mathrm{~m}, 1 \mathrm{H}) .{ }^{13} \mathrm{C} \mathrm{NMR}\left(\mathrm{CDCl}_{3}, 50 \mathrm{MHz}\right), \delta(\mathrm{ppm}): 11.6$, 13.5, 18.7, 27.2, 28.8, 56.2, 73.9, 109.5, 119.8, 123.6, 125.5, 126.6, 126.8, 127.8, 130.5, 133.2, 134.4, 140.4, 145.6. Diastereoisomer B: ${ }^{1} \mathrm{H}$ NMR $\left(\mathrm{CDCl}_{3}, 200 \mathrm{MHz}\right), \delta(\mathrm{ppm}): 0.7-0.95(\mathrm{~m}, 15 \mathrm{H}), 1.1-1.5(\mathrm{~m}, 12 \mathrm{H}), 2.52(\mathrm{~s}, 3 \mathrm{H})$, $2.98(\mathrm{~d}, 1 \mathrm{H}, J 6.2 \mathrm{~Hz}), 4.83\left(\mathrm{~d}, 1 \mathrm{H}, J 6.6 \mathrm{~Hz}\right.$, coupling with $\left.{ }^{119} \mathrm{Sn}{ }^{2} \mathrm{JSn}_{\mathrm{H}} 39 \mathrm{~Hz}\right), 5.50-5.60(\mathrm{~m}, 1 \mathrm{H}$, coupling with $\left.{ }^{119} \mathrm{Sn}^{3} \mathrm{~J}_{\mathrm{Sn}-\mathrm{H}} 8 \mathrm{~Hz}\right), 6.87-6.89(\mathrm{~m}, 2 \mathrm{H}), 6.99-7.31(\mathrm{~m}, 5 \mathrm{H}), 7.87-7.92(\mathrm{~m}, 1 \mathrm{H}) .{ }^{13} \mathrm{C} \mathrm{NMR}\left(\mathrm{CDCl}_{3}, 50 \mathrm{MHz}\right), \delta(\mathrm{ppm}):$ $11.1,13.5,27.2,28.8,53.9,74.1,109.8,119.5,123.5,125.8,126.1,126.7,127.8,130.9,134.2,134.5,139.2$, 145.3. MS (ESI, positive): $544.5[\mathrm{M}+\mathrm{H}]^{+}$. Elemental analysis: $\mathrm{C}_{27} \mathrm{H}_{41} \mathrm{~N}_{3} \mathrm{OSn}$ Calcd. C, 59.79; $\mathrm{H}, 7.62 ; \mathrm{N}, 7.75$. Found: C $59.90 \%, \mathrm{H} 7.56 \%, \mathrm{~N} 7.66$.

2-Benzotriazol-1-yl-1-thiophen-2-yl-2-tributylstannanyl-ethanol (12c). Following the general procedure GP4, silyl stannyl derivative 11 (121 mg, $0.25 \mathrm{mmol}$ ) and thiophene-2-carbaldehyde (29 mg, $0.29 \mathrm{mmol})$ gave, after purification, $12 \mathrm{c}$ as an orange oil $(97 \mathrm{mg}, 73 \%)$ as an equimolar mixture of diastereoisomers. Diastereoisomer $A:{ }^{1} \mathrm{H}$ NMR $\left(\mathrm{CDCl}_{3}, 200 \mathrm{MHz}\right), \delta(\mathrm{ppm}): 0.74-0.81(\mathrm{~m}, 9 \mathrm{H}), 0.88-0.95(\mathrm{~m}, 6 \mathrm{H}), 1.07-1.45(\mathrm{~m}, 12 \mathrm{H}), 3.06(\mathrm{~d}, 1 \mathrm{H}, J$ $6 \mathrm{~Hz}), 4.73(\mathrm{~d}, 1 \mathrm{H}, J 8.8 \mathrm{~Hz}), 5.73(\mathrm{dd}, 1 \mathrm{H}, J 8.8 \mathrm{~Hz}, 6 \mathrm{~Hz}), 6.93-6.97(\mathrm{~m}, 1 \mathrm{H}), 7.02-7.04(\mathrm{~m}, 1 \mathrm{H}), 7.18-7.26(\mathrm{~m}$, $2 \mathrm{H})$, 7.37-7.45 (m, 1H), 7.55-7.59 (m, 1H), 7.71-7.75 (m, 1H). $\left.{ }^{13} \mathrm{C} \mathrm{NMR} \mathrm{(CDCl}, 50 \mathrm{MHz}\right), \delta(\mathrm{ppm}): 10.7,13.5$, 27.2, 28.7, 56.5, 73.3, 110.3, 119.3, 123.7, 124.7, 125.2, 126.7, 126.8,134.4, 145.1, 145.2. Diastereoisomer B: ${ }^{1} \mathrm{H} \mathrm{NMR}\left(\mathrm{CDCl}_{3}, 200 \mathrm{MHz}\right), \delta(\mathrm{ppm}): 0.75-0.82(\mathrm{~m}, 9 \mathrm{H}), 0.84-0.92(\mathrm{~m}, 6 \mathrm{H}), 1.09-1.43(\mathrm{~m}, 12 \mathrm{H}), 3.41$ (bs, $\left.1 \mathrm{H}\right)$, $4.75(\mathrm{~d}, 1 \mathrm{H}, J 6 \mathrm{~Hz}), 5.61(\mathrm{ddd}, 1 \mathrm{H}, \mathrm{J} 1 \mathrm{~Hz}, 2.6 \mathrm{~Hz}, 6 \mathrm{~Hz}), 6.68-6.71(\mathrm{~m}, 1 \mathrm{H}), 6.78-6.83(\mathrm{~m}, 1 \mathrm{H}), 7.17-7.20(\mathrm{~m}$, $1 \mathrm{H})$, 7.24-7.40 (m, 3H), 7.94-7.99 (m, $1 \mathrm{H}) .{ }^{13} \mathrm{C} \mathrm{NMR}\left(\mathrm{CDCl}_{3}, 50 \mathrm{MHz}\right), \delta(\mathrm{ppm}):$ 11.3, 13.5, 27.2, 28.8, 57.5, 
73.7, 109.6, 119.7, 123.7, 124.0, 124.7, 126.8, 133.4, 145.4, 146.1. MS (ESI, positive): $536.3[\mathrm{M}+\mathrm{H}]^{+}$. Elemental analysis: $\mathrm{C}_{24} \mathrm{H}_{37} \mathrm{~N}_{3} \mathrm{OSSn}$ Calcd. C, 53.95; $\mathrm{H}, 6.98 ; \mathrm{N}, 7.86$. Found: $\mathrm{C} 53.71 \%, \mathrm{H} 7.14 \%, \mathrm{~N} 8.03$.

2-(1H-Benzo[d][1,2,3]triazol-1-yl)-1-phenylethan-1-ol (13a). Yellow oil. ${ }^{1} \mathrm{H} N M R\left(\mathrm{CDCl}_{3}, 200 \mathrm{MHz}\right), \delta(\mathrm{ppm})$ : $3.20(1 \mathrm{H}, \mathrm{bs}), 4.74(1 \mathrm{H}, \mathrm{dd}, J$ 8.0, $14.2 \mathrm{~Hz}), 4.84(1 \mathrm{H}, \mathrm{dd}, J 4.0,14.2 \mathrm{~Hz}), 5.38(1 \mathrm{H}, \mathrm{dd}, J$ J.0, $8.0 \mathrm{~Hz}), 7.30-7.51$ $(8 \mathrm{H}, \mathrm{m}), 7.94(1 \mathrm{H}$, ap d, $J 8.4 \mathrm{~Hz})$. Spectroscopic data matched those reported in the literature. ${ }^{48}$

2-(1H-Benzo[d][1,2,3]triazol-1-yl)-1-(o-tolyl)ethan-1-ol (13b). Yellow oil. ${ }^{1} \mathrm{H} \mathrm{NMR}\left(\mathrm{CDCl}_{3}, 200 \mathrm{MHz}\right), \delta$ (ppm): $2.38(3 \mathrm{H}, \mathrm{s}), 3.80(1 \mathrm{H}, \mathrm{bs}), 4.65(1 \mathrm{H}, \mathrm{dd}, J$ 8.6, $13.8 \mathrm{~Hz}), 4.74(1 \mathrm{H}, \mathrm{dd}, J 3.0,13.8 \mathrm{~Hz}), 5.55-5.61(1 \mathrm{H}, \mathrm{m}), 7.14-$ $7.30(\mathrm{~m}, 4 \mathrm{H}, \mathrm{m}), 7.42(1 \mathrm{H}, \mathrm{t}, J 7.4 \mathrm{~Hz}), 7.51(1 \mathrm{H}, \mathrm{d}, J 8.4 \mathrm{~Hz}), 7.63(1 \mathrm{H}, \mathrm{d}, J 6.8 \mathrm{~Hz}), 7.78(1 \mathrm{H}, \mathrm{d}, J 8.4 \mathrm{~Hz})$. Spectroscopic data matched those reported in the literature. ${ }^{49}$

\section{Acknowledgements}

We thank MIUR-Italy (“Progetto Dipartimenti di Eccellenza 2018-2022" allocated to Department of Chemistry "Ugo Schiff").

\section{References}

1. Organosilicon Compounds. Theory and Experiment (Synthesis) Lee, V. Y., Ed.; Academic Press, Elsevier: London, 2017; Vol. 1.

2. Xu, L.-W. Curr. Org. Chem. 2011, 15, 2742.

https://doi.org/10.2174/138527211796378460

3. Gawronski, J.; Wascinska, N.; Gajewy, J. Chem. Rev. 2008, 108, 5227-5252 and references cited therein. https://doi.org/10.1021/cr800421c

4. Hiyama, T.; Shirakawa, E. Topics in Current Chemistry 2002, 219, 61-85. https://doi.org/10.1007/3-540-45313-X 3

5. Ager, D. J. Chem. Soc., Perkin Trans. 1 1986, 195-204. https://doi.org/10.1039/P19860000195

6. Ager, D. J.; Cooke, G. E.; East, M. B.; Mole, S. J.; Rampersaud, A.; Webb, V. J. Organometallics 1986, 5, 1906-1908.

https://doi.org/10.1021/om00140a026

7. Davis, D. D.; Gray, C. E. J. Org. Chem. 1970, 35, 1303-1307. https://doi.org/10.1021/j000830a011

8. Kauffmann, T.; Kriegesmann, R.; Hamsen, A. Chem. Ber. 1982, 115, 1818-1824. https://doi.org/10.1002/cber.19821150515

9. Kawashima, T.; Iwama, N.; Okazaki, R. J. Am. Chem. Soc. 1993, 115, 2507-2508. https://doi.org/10.1021/ja00059a056

10. Cuadrado, P.; González-Nogal, A. Tetrahedron Lett. 2001, 42, 8993-8996. https://doi.org/10.1016/S0040-4039(01)01990-6

11. Yoshida, J.-i.; Watanabe, M.; Toshioka, H.; Imagawa, M.; Suga, S. J. Electroanal. Chem. 2001, 507, 55-65. https://doi.org/10.1016/S0022-0728(01)00402-8

12. Degl'Innocenti, A.; Pollicino, S.; Capperucci, A. Chem. Commun. 2006, 4881-4893 and references cited therein. 
https://doi.org/10.1039/b608816n

13. Degl'Innocenti, A.; Capperucci, A. Eur. J. Org. Chem. 2000, 2171-2186 and references cited therein. https://doi.org/10.1002/1099-0690(200006)2000:12<2171::AID-EJOC2171>3.0.CO;2-3

14. Capperucci, A.; Degl'Innocenti, A.; Nocentini, T.; Pollicino, S. J. Sulfur Chem. 2009, 30, 319-326. https://doi.org/10.1080/17415990902998587

15. Degl'Innocenti, A.; Capperucci, A.; Nocentini, T. Tetrahedron Lett. 2001, 42, 4557-4559. https://doi.org/10.1016/S0040-4039(01)00771-7

16. Lenardão, E. J.; Santi, C.; Sancineto, L. New Frontiers in Organoselenium Compounds, Springer, New York, 2018.

https://doi.org/10.1007/978-3-319-92405-2

17. Liao, L.; Guo, R.; Zhao, X. Angew. Chem. Int. Ed. 2017, 56, 3201. https://doi.org/10.1002/anie.201610657

18. Mukherjee, A. J.; Zade, S. S.; Singh, H. B.; Sunoj, R. B. Chem. Rev. 2010, 110, 4357-4416. https://doi.org/10.1021/cr900352i

19. Tanini, D.; Scarpelli, S.; Ermini, E.; Capperucci, A. Adv. Synth. Catal., 2019, 361, 2337-2346. https://doi.org/10.1002/adsc.201900168

20. Patra, A. R.; Roy, S. S.; Basu, A.; Bhuniya, A.; Bhattachariee, A.; Hajra, S.; Sk, U. H.; Baral, R.; Bhattacharya, S. Sci. Rep. 2018, 8, 2194-2206. https://doi.org/10.1038/s41598-018-19854-5

21. Nogueira, C. W.; Zeni, G.; Rocha, J. B. T. Chem. Rev. 2004, 104, 6255-6286. https://doi.org/10.1021/cr0406559

22. For book chapters see: Singh, F. V.; Wirth, T. in Organoselenium Compounds in Biology and Medicine: Synthesis, Biological and Therapeutic Treatments Eds. Jain, V. K.; Priyadarsini, K. I. Royal Society of Chemistry: London, 2018, Chapter 3 and references cited therein; Carrol, L. D.; Davies, M. J. in Organoselenium Compounds in Biology and Medicine: Synthesis, Biological and Therapeutic Treatments (Eds. Jain, V. K.; Priyadarsini, K. I.) Royal Society of Chemistry: London, 2018, Chapter 9 and references cited therein.

23. Tanini, D.; Lupori, B.; Malevolti, G.; Ambrosi, M.; Lo Nostro, P.; Capperucci, A. Chem. Commun. 2019, 55, 5705-5708.

https://doi.org/10.1039/C9CC02427A

24. Satheeshkumar, K.; Raju, S.; Singh, H. B.; Butcher, R. J. Chem. Eur. J. 2018, 24, 17513. https://doi.org/10.1002/chem.201803776

25. Tanini, D.; Grechi, A.; Ricci, L.; Dei, S.; Teodori, E.; Capperucci, A. New J. Chem. 2018, 42, 6077-6083. https://doi.org/10.1039/C8NJ00700D

26. Rocha, J. B. T.; Oliveir, C. S.; Nogara, P. A. in Organoselenium Compounds in Biology and Medicine: Synthesis, Biological and Therapeutic Treatments Eds. Jain, V. K.; Priyadarsini, K. I. Royal Society of Chemistry: London, 2018, Chapter 13.

27. Angeli, A.; Tanini, D.; Nocentini, A.; Capperucci, A.; Ferraroni, M.; Gratteri, P.; Supuran, C. T. Chem. Commun. 2019, 55, 648-651.

https://doi.org/10.1039/C8CC08562E

28. Krasowska, D.; Iraci, N.; Santi, C.; Drabowicz, J.; Cieslak, M., Kaźmierczak-Barańska, J.; Palomba, M.; Królewska-Golińska, K., Magiera, J.; Sancineto, L. Molecules 2019, $24,2914$. https://doi.org/10.3390/molecules24162914

29. Pang, Y.; An, B.; Lou, L.; Zhang, J., Yan, J.; Huang, L.; Li, X.; Yin, S. J. Med. Chem. 2017, 60, 7300-7314. 
https://doi.org/10.1021/acs.jmedchem.7b00480

30. Rizvia, M. A.; Guru, S.; Naqvi, T.; Kumar, M.; Kumbhar, N.; Akhoon, S.; Banday, S.; Singh, S. K.; Bhushan, S.; Peerzada, M.; Shah, B. A. Bioorg. Med. Chem. Lett. 2014, 24 , 3440-3446.

https://doi.org/10.1016/j.bmcl.2014.05.075

31. Tanini, D.; Capperucci, A.; Supuran, C. T.; Angeli, A. Bioorg. Chem. 2019, 87, 516-522.

https://doi.org/10.1016/i.bioorg.2019.03.062

32. Kostic, M. D.; Divac, V. M. Environ. Chem. Lett. 2019, 17, 897-915 and references cited therein. https://doi.org/10.1007/s10311-018-00848-8

33. Tanini, D.; Capperucci, A. New J. Chem. 2019, 43, 11451-11468.

https://doi.org/10.1039/C9NJ02320H

34. Tanini, D.; Tiberi, C.; Gellini, C.; Salvi, P. R.; Capperucci, A. Adv. Synth. Catal. 2018, 360, 3367-3375. https://doi.org/10.1002/adsc.201800602

35. Tanini, D.; Borgogni C.; Capperucci, A. New J. Chem. 2019, 43, 6388-6393.

https://doi.org/10.1039/C9NJ00657E

36. Krief, A.; Dumont, W.; Gillina, F. Arkivoc 2007, (vii) 51-64.

37. Lenardão, E. J.; Cella, R.; Jacob, R. G., da Silva, T. B.; Perin, G. J. Braz. Chem. Soc. 2006, 17, 1031-1038. https://doi.org/10.1590/S0103-50532006000500031

38. Recchi, A. M. S.; Back, D. F.; Zeni, G. J. Org. Chem. 2017, 82, 2713-2723. https://doi.org/10.1021/acs.joc.7b00050

39. Senatore, R.; Castoldi, R.; lelo, L.; Holzer, W.; Pace, V. Org. Lett. 2018, 20, 2685-2688. https://doi.org/10.1021/acs.orglett.8b00896

40. Capperucci, A.; Degl'Innocenti, A.; Pollicino, S.; Acciai, M.; Castagnoli, G., Malesci, I.; Tiberi, C. Heteroatom Chem. 2007, 18, 516-526.

https://doi.org/10.1002/hc.20345

41. Katritzky, A. R.; Rachwal, S. Chem. Rev. 2011, 111, 7063-7120 and references cited therein. https://doi.org/10.1021/cr200031r

42. Katritzky, A. R.; Rogovoy, B. V. Chem. Eur. J. 2003, 9, 4586-4593 and references cited therein. https://doi.org/10.1002/chem.200304990

43. Wang, Z-L.; Tang, R-Y.; Luo, P-S.; Deng, C-L.; Zhong, P.; Li, J-H. Tetrahedron 2008, 64, 10670-10675. https://doi.org/10.1016/j.tet.2008.09.022

44. Li, J.; Liu, G.; Shi, L.; Xing, Q.; Li, F. Green Chem. 2017,19, 5782-5788.

https://doi.org/10.1039/C7GC02335A

45. Choudhuri, K.; Mandal, A.; Mal, P. Chem. Commun. 2018, 54, 3759-3762. https://doi.org/10.1039/C8CC01359D

46. Zhao, H.; Jiang, Y.; Chen, Q.; Cai, M. New J. Chem. 2015, 39, 2106-2115. https://doi.org/10.1039/C4NJ01687D

47. Leng, T.; Wu, G.; Zhou, Y-B.; Gao, W.; Ding, J.; Huang, X.; Liu, M.; Wu, H. Adv. Synth. Catal. 2018, 360, 43364340.

https://doi.org/10.1002/adsc.201800896

48. Xue, Q.; Xie, J.; Xu, P.; Hu, K.; Cheng, Y.; Zhu, C. ACS Catal. 2013, 3, 1365-1368.

https://doi.org/10.1021/cs400250m

49. Xiao, X.; Lin, D.; Tong, S.; Mo, H. Synlett 2011, 19, 2823-2826.

https://doi.org/10.1055/s-0031-1289555 